\begin{tabular}{|c|l|}
\hline Title & MASS SUPPLY TO GA LA CTIC CENTER DUE TO NESTED BA RS IN THE GALAXY \\
\hline Author(s) & Namekata, Daisuke; Habe, A sao; Matsui, Hidenori; Saitoh, Takay uki R. \\
\hline Citation & $\begin{array}{l}\text { The A strophysical Journal, 691/2), 1525_1539 } \\
\text { https://doi.org/10.1088/0004637X /691/2/1525 }\end{array}$ \\
\hline Issue Date & 2009-02-01 \\
\hline Doc URL & http://hdl.handle.net/2115/48499 \\
\hline Rights & Published under licence in [The A strophysical Journal] by IOP Publishing Ltd. \\
\hline Type & article \\
\hline File Information & apj_691_2_1525.pdf \\
\hline
\end{tabular}

Instructions for use 


\title{
MASS SUPPLY TO GALACTIC CENTER DUE TO NESTED BARS IN THE GALAXY
}

\author{
Daisuke Namekata ${ }^{1}$, Asao Habe ${ }^{1}$, Hidenori Matsui ${ }^{2}$, and Takayuki R. Saitoh ${ }^{3}$ \\ ${ }^{1}$ Department of Cosmosciences, Graduate School of Science, Hokkaido University, Sapporo 060-0810, Japan; name@ astro1.sci.hokudai.ac.jp, \\ habe@astro1.sci.hokudai.ac.jp \\ ${ }^{2}$ Division of Theoretical Astronomy, National Astronomical Observatory of Japan, 2-21-1, Osawa, Mitaka, Tokyo 181-8588, Japan; hidenori.matsui@nao.ac.jp \\ ${ }^{3}$ Center for Computational Astrophysics, National Astronomical Observatory of Japan, 2-21-1, Osawa, Mitaka, Tokyo 181-8588, Japan; saitoh.takayuki@nao.ac.jp \\ Received 2008 July 22; accepted 2008 October 6; published 2009 February 5
}

\begin{abstract}
We investigate a rapid mass supply process by nested bars in the Galaxy by numerical simulation. We simulate gas flow in the whole Galaxy disk with nested bars, which are the outer bar and the inner bar, with a high spatial resolution in the Galactic central region. We assume two cases of the inner bar size: one smaller and one larger than the radius of the $200 \mathrm{pc}$ gas ring which corresponds to the central molecular zone. From our numerical results, in the large size bar cases, the inner bars with large elongation induce sufficient mass inflow and destroy the $200 \mathrm{pc}$ gas ring. On the other hand, in the small size bar cases, the inner bars with large elongation induce large mass inflow and do not destroy the $200 \mathrm{pc}$ gas ring. This mass inflow is caused by straight shocks excited by the inner bar. In this case, nuclear gas disks of $\sim 15 \mathrm{pc}$ radius are formed. The nuclear gas disks are self-gravitationally unstable and we expect the formation of compact star clusters under a strong tidal force in the nuclear gas disks. We discuss the evolution of the nuclear gas disk.
\end{abstract}

Key words: Galaxy: center - Galaxy: kinematics and dynamics - methods: numerical

Online-only material: color figure

\section{INTRODUCTION}

Gas fueling to a galactic center is very important for the activity of active galactic nuclei (AGNs), the growth of supermassive black holes (SMBHs), nuclear starbursts, the formation of super star clusters in a galactic central region, and other interesting phenomena. Our Galaxy is very interesting for this study because of the following reasons. First, the center of our Galaxy is the closest galactic center. Its distance is about $8.0 \mathrm{kpc}$ away (Eisenhauer et al. 2003). Therefore, there are many observational data with high resolution over wide wavelength. It is easy to compare those with numerical simulations. Second, there is evidence of recent mass supply in our Galactic center. There are young massive compact star clusters (the Arches, Quintuplet, and Central clusters) in the Galactic center. These clusters are located within $30 \mathrm{pc}$ from the Galactic center and have a number of OB stars (Figer 2003). Because of the short age of the young stars, we can determine that a massive star formation occurred within the last several million years in the central region of the Galaxy (Mezger et al. 1996). Formation of these clusters requires a large amount of gas. The circumnuclear gas disk (CND), which is dense $\left(10^{5} \mathrm{~cm}^{-3}\right)$, clumpy, and turbulent with large line widths $\left(\geqslant 40 \mathrm{~km} \mathrm{~s}^{-1}\right.$; Coil \& Ho 1999), has a radius of a few parsecs and a mass of $\approx 10^{6} M_{\odot}$ (Christopher et al. 2005). Coil \& Ho (1999) found a gas stream from the giant molecular cloud (GMC; the $20 \mathrm{~km} \mathrm{~s}^{-1}$ cloud) near the Galactic center to the CND. This may be gas inflow to the CND.

It is expected that a vast amount of gas is supplied from the central molecular zone (CMZ), which is a ring-like gas distribution and extends over the range of Galactic longitude $-1.5 \leqslant l \leqslant 2^{\circ}$, to the Galactic center (Serabyn \& Morris 1995; Morris \& Serabyn 1996). The size of the CMZ is $\sim 200 \mathrm{pc}$. It is certainly formed by the large-scale bar (Binney et al. 1991; Morris \& Serabyn 1996; Sawada et al. 2004) and has a large amount of molecular gas $(5-10) \times 10^{7} M_{\odot}$ (Serabyn \& Morris 1995). However, it is unclear how the gas is transported further in. Secondary effects, such as dissipation, gravitational instability, and magnetic viscosity, can then drive the gas further in but at a slower rate (e.g., Heller et al. 2001; Fukuda et al. 2000; Morris \& Serabyn 1996).

For the gas feeding, many authors show that a bar has an important role (e.g., Athanassoula 1992). Nested bars, which consist of an outer bar and inner bars, may play an important role in the gas feeding to galactic centers. This idea was first proposed by Shlosman et al. (1989) as a mechanism for fueling AGNs. Inspired by the idea of Shlosman et al. (1989), many numerical studies have been performed (Friedli \& Martinet 1993; Friedli 1996; Maciejewski \& Sparke 1997, 2000; Heller et al. 2001, 2006; Shlosman \& Heller 2002b; Rautiainen et al. 2002; Maciejewski et al. 2002; El-Zant \& Shlosman 2003; Englmaier \& Shlosman 2004; Debattista \& Shen 2007; Shen \& Dibattista 2007). Friedli \& Martinet (1993) performed threedimensional simulations of gas and stars and showed that an inner bar can drive the gas infall to a galactic center.

Nested bars are observed in nearby barred galaxies in a large fraction ( 30\%; Wozniak et al. 1995; Friedli et al. 1996; Jungwiert et al. 1997; Erwin \& Sparke 2002; Erwin 2004). The large fraction indicates that nested bars are dynamically stable or recurrent structures. Nested bars are expected to be dynamically decoupling, since the orientations of both bars are random (Buta \& Crocker 1993). Dynamical decoupling of these was also reported in many numerical studies (Friedli \& Martinet 1993; Maciejewski \& Sparke 2000; Rautiainen et al. 2002; Englmaier \& Shlosman 2004; Debattista \& Shen 2007).

An increasing number of observational studies show the effect of nested bars in gas flows in central regions of galaxies. Fathi et al. (2006) observed the central region of the doublebarred galaxy, NGC 1097, with high resolution, using Gemini Multi-Object Spectrograph-Integral Field Unit (GMOS-IFU) and Hubble Space Telescope-ACS. They show clear evidence of radial streaming motion down to about $10 \mathrm{pc}$ from the nucleus by mapping the gas velocity fields. Schinnerer et al. $(2006,2007)$ 

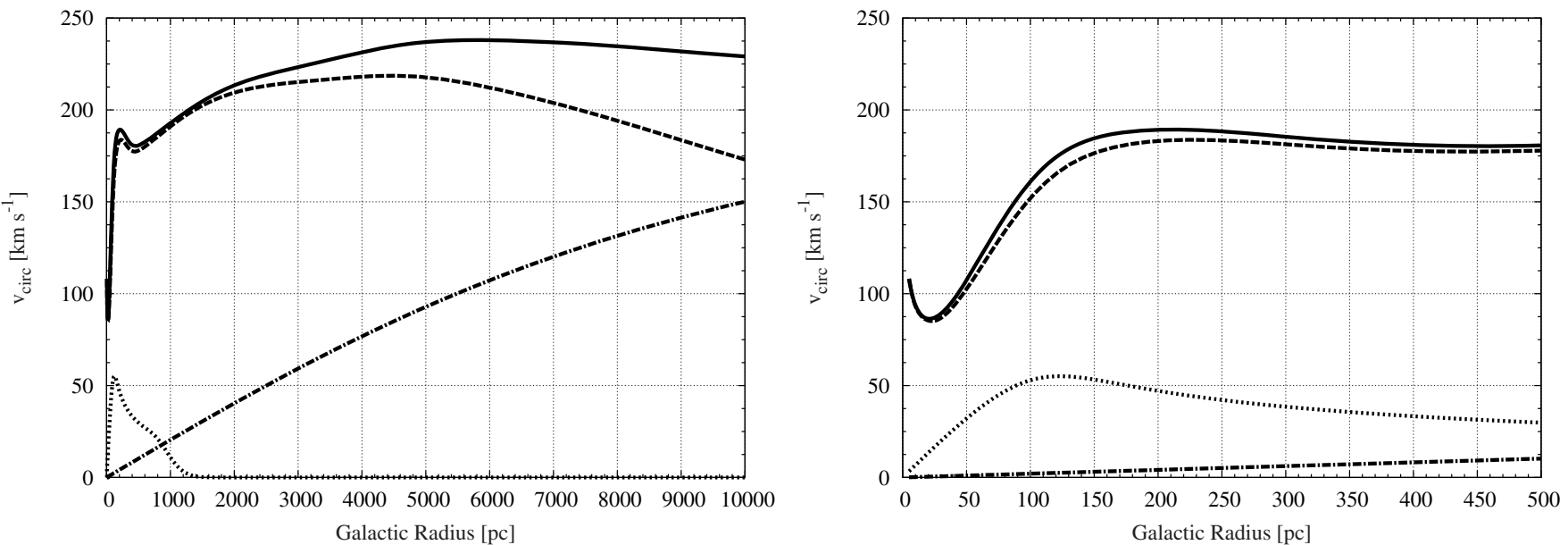

Figure 1. Rotation curves in the entire (left panel) and the central region (right panel) of the model S33. These lines show rotation curves by total mass (solid line), disk and bulge (dashed line), inner bar (dotted line), and dark halo (dotted-dashed line), respectively.

observed molecular emissions in the central region of the nearby double-barred spiral galaxy NGC 6946 with a very high spacial resolution $\left(\lesssim 1^{\prime \prime}\right)$ with the IRAM Plateau de Bure interferometer. They showed that there are nuclear massive gas clumps and straight dust lanes inside the inner bar. They concluded that the inner bar is closely related with the pile-up of molecular gas to the nucleus. Meier et al. (2008) observed the central region of the barred galaxy, Maffei 2, with Berkeley-IllinoisMaryland Association (BIMA) and the Owens Valley Radio Observatory (OVRO). They show a nuclear ring, whose radius is $\sim 80 \mathrm{pc}$ and mass is $6.9 \times 10^{6} M_{\odot}$, well inside the bar and that overall morphology of the gas, including the nuclear ring, can be explained by a nuclear bar by comparing the position-velocity $(p-v)$ diagram of the molecular gas with orbits of molecular clouds in their nuclear bar model. These studies support the important role of inner bars in transporting gas to a galactic center.

Recently, observational studies show evidence of an inner bar in our Galaxy, which is much smaller than the outer bar of semimajor axis $3.5 \mathrm{kpc}$. Alard (2001) studied the surface density of the old stellar population in the inner bulge by using the Two Micron All Sky Survey data and showing evidence of the inner bar. Nishiyama et al. $(2005,2006)$ investigated the shift of the peak position of red clump stars' distribution over $|l|<10.5$ using the IRAF $1.4 \mathrm{~m}$ telescope with the near-infrared camera SIRIUS and showed that the gradient of this shift clearly changes in $|l|<4^{\circ}$. They interpreted that this structure may be due to the inner bar.

We study the possibility that the inner bar plays an important role in the mass supply from the CMZ to the Galactic center. Previous theoretical studies have not reported the case of a large contribution of inner bars in gas supply to a galactic center (e.g., Maciejewski et al. 2002; Rautiainen et al. 2002). They studied limited cases. We investigate various inner bar models in this paper. We perform two-dimensional hydrodynamical simulations in a gravitational potential model of our Galaxy, assuming several inner bar models. In the simulations, we systematically change the mass and the axial ratio of inner bar models, since parameters of the inner bar are not clear from observations.

In Section 2, we give our gravitational models and numerical method. In Section 3, we show the results of our simulations. In Section 4 , we discuss the gravitational instability and evolution of nuclear gas disks, which are obtained in our numerical results. In Section 5, we summarize our study.

\section{MODEL}

\subsection{Gravitational Potential of the Galaxy}

As the gravitational potential of our Galaxy except for an inner bar, we assume the model of Bissantz et al. (2003) for the Galactic bulge, the stellar disk, the outer bar, the spiral arms, and the dark halo $(R>500 \mathrm{pc})$, and Launhardt et al. (2002) for the nuclear bulge $(R<500 \mathrm{pc})$ and the SMBH. Bissantz et al. (2003) simulated gas motion in our Galaxy potential model, which consists of the Galactic bulge, the stellar disk, the outer bar, the spiral arms, and the dark halo. They gave pattern speeds of the outer bar and the spiral arms ( $\Omega_{\mathrm{OB}} \approx 60 \mathrm{Gyr}^{-1}$ and $\Omega_{\mathrm{SP}} \approx 20 \mathrm{Gyr}^{-1}$, respectively) to reproduce observational gas kinematics of molecular clouds. Launhardt et al. (2002) analyzed IRAS and COBE DIRBE data of the central $500 \mathrm{pc}$ of our Galaxy. They gave mass distribution of the nuclear bulge, which is distinguished from the Galactic bulge by its flat disk-like feature, assuming a constant mass-to-light ratio. They estimated that the nuclear bulge has a mass of $(1.4 \pm 0.6) \times 10^{9} M_{\odot}$. We assume the rotation curve obtained from the mass distribution of the nuclear bulge and the SMBH in $R \leqslant 500 \mathrm{pc}$ for the rotation curve of the total stellar mass. We smoothly connect the rotation curves obtained from the nuclear bulge and the SMBH in $R \leqslant 500 \mathrm{pc}$ and from the stellar component of Bissantz et al. (2003) in $R>500 \mathrm{pc}$. Details on the gravitational potential of the outer bar, the spiral arms, and the dark halo are described in Bissantz et al. (2003). Figure 1 shows the rotation curve of one of our models, the model S33. In this figure, we use the axially averaged mass distribution of the inner and outer bars. In Figure 2, we show the angular velocity curve of the model S33. In this figure, there is the local maximum of $\Omega-\kappa / 2$ at $150 \mathrm{pc}$, where $\Omega$ is the angular velocity and $\kappa$ is the epicyclic frequency. We point out that the curve of $\Omega-\kappa / 2$ in $R<500 \mathrm{pc}$ is rather uncertain, since it is difficult to measure accurately the mass profile in this scale.

\subsection{Inner Bar Potential}

We assume a Ferrers bar models for inner bars, since a density profile of the inner bar is not observationally confirmed. The 

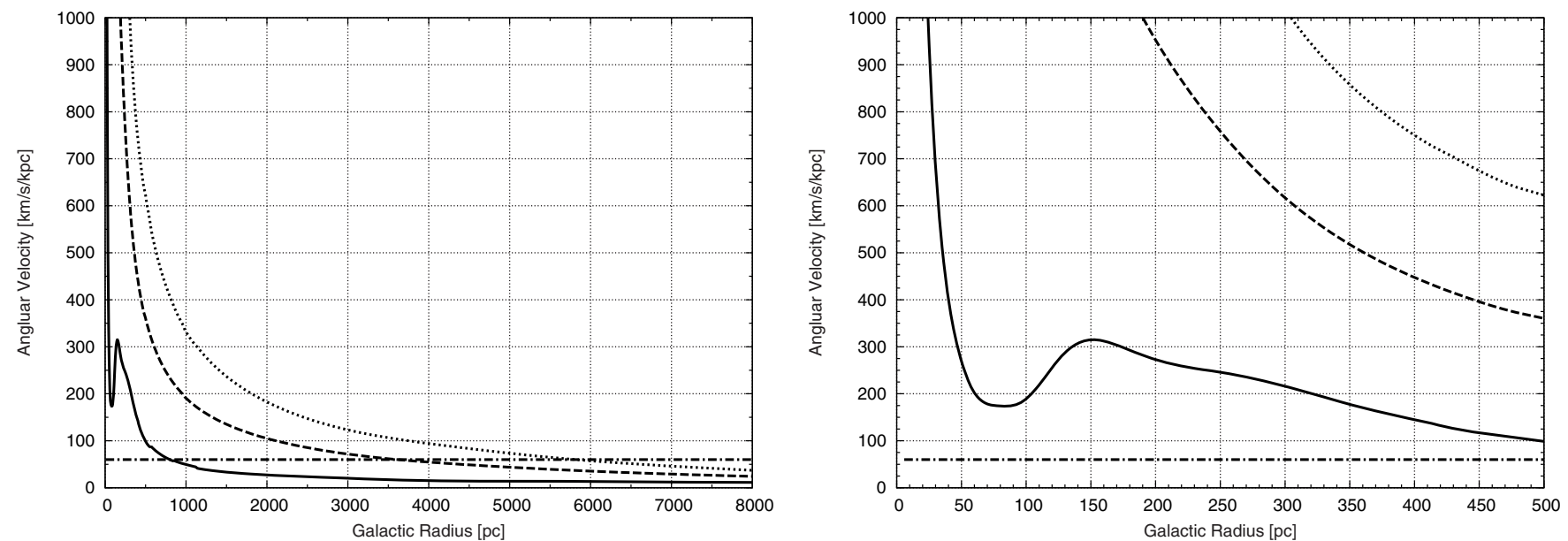

Figure 2. Angular frequency curve in the entire region of the Galaxy (left) and the central region of the Galaxy (right). These lines show angular frequency curves of $\Omega-\kappa / 2$ (solid line), $\Omega$ (dashed line), and $\Omega+\kappa / 2$ (dotted line), and the pattern speed of the outer bar $\Omega_{\mathrm{OB}}$ (dash-dotted line). The positions of the inner Lindblad resonance $\left(R_{\mathrm{ILR}}\right)$, the corotation resonance $\left(R_{\mathrm{CR}}\right)$, and the outer Lindblad resonance $\left(R_{\mathrm{OLR}}\right)$ of the outer bar are $750 \mathrm{pc}, 3750 \mathrm{pc}$, and $6750 \mathrm{pc}$, respectively.

Table 1

Axial Ratio and Mass of the Small Inner Bar Models

\begin{tabular}{lccccc}
\hline \hline$a_{\mathrm{IB}} / b_{\mathrm{IB}}{ }^{\mathrm{a}}$ & \multicolumn{5}{c}{$M_{\mathrm{IB}}\left[M_{\odot}\right]^{\mathrm{b}}$} \\
\cline { 2 - 6 } & $5.0 \times 10^{7}$ & $7.5 \times 10^{7}$ & $1.0 \times 10^{8}$ & $1.5 \times 10^{8}$ & $2.5 \times 10^{8}$ \\
\hline 4 & $\mathrm{~S} 41$ & $\mathrm{~S} 42$ & $\mathrm{~S} 43$ & $\cdots$ & $\cdots$ \\
& $(0.115)^{\mathrm{c}}$ & $(0.168)$ & $(0.218)$ & & \\
3 & $\mathrm{~S} 31$ & $\mathrm{~S} 32$ & $\mathrm{~S} 33$ & $\mathrm{~S} 34$ & $\mathrm{~S} 35$ \\
& $(0.062)$ & $(0.092)$ & $(0.121)$ & $(0.175)$ & $(0.274)$ \\
2 & $\mathrm{~S} 21$ & $\mathrm{~S} 22$ & $\mathrm{~S} 23$ & $\mathrm{~S} 24$ & $\mathrm{~S} 25$ \\
& $(0.024)$ & $(0.036)$ & $(0.048)$ & $(0.071)$ & $(0.115)$ \\
$4 / 3$ & $\cdots$ & $\cdots$ & $\mathrm{S} 13$ & $\mathrm{~S} 14$ & $\mathrm{~S} 15$ \\
& & & $(0.012)$ & $(0.019)$ & $(0.031)$
\end{tabular}

Notes.

${ }^{\text {a }}$ The axial ratio of the inner bar model. $a_{\mathrm{IB}}$ is a semimajor axis of the inner bar model and $b_{\mathrm{IB}}$ is a semiminor axis of the inner bar model.

$\mathrm{b}$ The mass of the inner bar model.

${ }^{\mathrm{c}}$ The values in parentheses show $Q_{T}$.

Ferrers bar model has a density distribution of

$$
\rho(x, y, z)=\rho_{0}\left(1-\frac{x^{2}}{a^{2}}-\frac{y^{2}}{b^{2}}-\frac{z^{2}}{c^{2}}\right)^{n},
$$

where $\rho_{0}$ is the density at the origin (Ferrers 1877). We assume $n=1$ and $b=c . \rho_{0}$ is related to the mass of the inner bar $M_{\mathrm{IB}}$ through $\rho_{0}=\frac{15 M_{\mathrm{IB}}}{8 \pi a b^{2}}$ for $n=1$. Parameters we choose are given in Tables 1 and 2 .

We assume two cases of the length of the semimajor axis of the inner bar models, $a_{\mathrm{IB}}=200 \mathrm{pc}$ and $600 \mathrm{pc}$ from the following studies. Wozniak et al. (1995) performed the BVRI survey of 36 disk galaxies selected as candidates for having an inner bar or a triaxial bulge within the outer bar. They showed that outer to inner bar axis ratios, $a_{\mathrm{OB}} / a_{\mathrm{IB}}$, are in the range of 3.7-18.0 with the mean value of 7.2. Friedli et al. (1996) observed 13 disk galaxies, which had been classified into galaxies likely having an inner bar or a triaxial bulge within the outer bar in Wozniak et al. (1995), with $J H K$ band. They show a similar result, $4.0 \leqslant a_{\mathrm{OB}} / a_{\mathrm{IB}} \leqslant 13.4$, with the mean value of 7.2. In our Galaxy, the above ranges correspond to $a_{\mathrm{IB}}=200-875 \mathrm{pc}$ for the semimajor axis of the outer bar, $3.5 \mathrm{kpc}$. If our Galaxy is a normal nested barred galaxy, our assumed values of $a_{\mathrm{IB}}$ are in this range.
Table 2

Axial Ratio and Mass of the Large Inner Bar Models

\begin{tabular}{lccccc}
\hline \hline$a_{\mathrm{IB}} / b_{\mathrm{IB}}{ }^{\mathrm{a}}$ & \multicolumn{5}{c}{$M_{\mathrm{IB}}\left[M_{\odot}\right]^{\mathrm{b}}$} \\
\cline { 2 - 6 } & $1.0 \times 10^{8}$ & $2.5 \times 10^{8}$ & $5.0 \times 10^{8}$ & $7.5 \times 10^{8}$ & $1.0 \times 10^{9}$ \\
\hline 4 & $\mathrm{~L} 41$ & $\mathrm{~L} 42$ & $\mathrm{~L} 43$ & $\cdots$ & $\cdots$ \\
& $(0.047)^{\mathrm{c}}$ & $(0.115)$ & $(0.220)$ & & \\
3 & $\mathrm{~L} 31$ & $\mathrm{~L} 32$ & $\mathrm{~L} 33$ & $\mathrm{~L} 34$ & $\mathrm{~L} 35$ \\
& $(0.031)$ & $(0.075)$ & $(0.146)$ & $(0.213)$ & $(0.276)$ \\
2 & $\cdots$ & $\mathrm{L} 22$ & $\mathrm{~L} 23$ & $\mathrm{~L} 24$ & $\mathrm{~L} 25$ \\
& $\ldots$ & $(0.036)$ & $(0.071)$ & $(0.105)$ & $(0.138)$ \\
$4 / 3$ & $\cdots$ & $\mathrm{L} 13$ & $\mathrm{~L} 14$ & $\mathrm{~L} 15$ \\
& $\cdots$ & & $(0.023)$ & $(0.036)$ & $(0.046)$ \\
& & & & &
\end{tabular}

Notes.

${ }^{\text {a }}$ The axial ratio of the inner bar model. $a_{\mathrm{IB}}$ is a semimajor axis of the inner bar model and $b_{\mathrm{IB}}$ is a semiminor axis of the inner bar model.

${ }^{b}$ The mass of the inner bar model.

c The values in parentheses show $Q_{T}$.

In our assumption on sizes of the inner bar models, we also consider the fact that inner bars often coexist with nuclear rings (Buta \& Crocker 1993; Shaw et al. 1993; Erwin \& Sparke 2002). Erwin \& Sparke (2002) found that $60 \%$ of their sample galaxies with nuclear rings have inner bars. In such galaxies, inner bars are often surrounded by nuclear rings and the size of the inner bars is comparable with that of the nuclear rings. In our Galaxy, if the CMZ corresponds to such a nuclear ring, the size of the inner bar may be comparable with the size of the CMZ $(R \approx 200 \mathrm{pc})$. This is consistent with the projected size of the inner bar of Alard (2001) that is $\sim 1.5-2^{\circ} \approx 200-300$ pc. However, the size of the inner bar proposed by Nishiyama et al. $(2005,2006)$ is $\approx 520 \mathrm{pc}$, and is much larger than the size of the CMZ.

Our assumption on sizes of the inner bar models is consistent with recent numerical simulations. Debattista \& Shen (2007) and Shen \& Dibattista (2007) investigated the formation of a long-lived inner bar from a pseudobulge by performing $\mathrm{N}$-body simulations. They showed that inner bar ends are much smaller than their corotation radius $R_{\mathrm{CR}}$. A similar result was also obtained in Friedli \& Martinet (1993). The $R_{\mathrm{CR}}$ of the inner bar is as large as $600 \mathrm{pc}$ in our models, if the pattern speed of the inner bar is near the local maximum of $\Omega-\kappa / 2$ (our choice is intended to be consistent with the $N$-body simulations; see 
Table 3

Pattern Speeds of the Inner Bar Models

\begin{tabular}{|c|c|c|c|c|c|c|c|c|c|c|c|c|c|c|c|c|c|}
\hline \multirow[t]{2}{*}{ Model Name } & \multicolumn{17}{|c|}{ Pattern Speed of the Inner Bar $\Omega_{\mathrm{IB}}\left(\mathrm{km} \mathrm{s}^{-1} \mathrm{kpc}^{-1}\right)$} \\
\hline & 175 & 200 & 225 & 230 & 240 & 250 & 260 & 270 & 275 & 280 & 290 & 300 & 310 & 320 & 325 & 350 & 375 \\
\hline S41 & . & $\bigcirc^{a}$ & $\cdots$ & $\bigcirc$ & $\bigcirc$ & $\bigcirc$ & $\bigcirc$ & $\bigcirc$ & $\cdots$ & $\bigcirc$ & $\bigcirc$ & 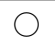 & & $\bigcirc$ & $\cdots$ & $\cdots$ & $\cdots$ \\
\hline $\mathrm{S} 42$ & $\bigcirc$ & $\bigcirc$ & $\bigcirc$ & $\cdots$ & $\cdots$ & $\bigcirc$ & $\cdots$ & $\cdots$ & $\bigcirc$ & $\cdots$ & $\bigcirc$ & & & $\cdots$ & $\bigcirc$ & $\bigcirc$ & $\bigcirc$ \\
\hline $\mathrm{S} 43$ & $\bigcirc$ & $\bigcirc$ & $\bigcirc$ & $\ldots$ & $\ldots$ & $\bigcirc$ & $\ldots$ & $\ldots$ & $\bigcirc$ & $\ldots$ & $\bigcirc$ & & & $\ldots$ & 0 & $\bigcirc$ & $\bigcirc$ \\
\hline $\mathrm{S} 31$ & $\cdots$ & $\cdots$ & $\ldots$ & $\cdots$ & $\cdots$ & $\cdots$ & $\cdots$ & $\cdots$ & $\cdots$ & $\cdots$ & $\cdots$ & & $\cdots$ & $\cdots$ & $\cdots$ & $\cdots$ & $\cdots$ \\
\hline $\mathrm{S} 32$ & $\bigcirc$ & $\bigcirc$ & $\bigcirc$ & $\cdots$ & $\cdots$ & & $\bigcirc$ & $\bigcirc$ & $\cdots$ & $\bigcirc$ & $\bigcirc$ & & & $\bigcirc$ & $\cdots$ & $\cdots$ & $\cdots$ \\
\hline S33 & $\bigcirc$ & $\bigcirc$ & $\bigcirc$ & $\cdots$ & $\cdots$ & & $\cdots$ & $\cdots$ & $\bigcirc$ & $\cdots$ & $\bigcirc$ & & & $\cdots$ & $\bigcirc$ & $\bigcirc$ & . \\
\hline S34 & $\cdots$ & $\cdots$ & $\cdots$ & $\cdots$ & $\cdots$ & $\bigcirc$ & $\bigcirc$ & $\bigcirc$ & $\cdots$ & $\bigcirc$ & $\bigcirc$ & & & $\ldots$ & $\ldots$ & $\ldots$ & $\cdots$ \\
\hline S35 & $\ldots$ & $\ldots$ & $\ldots$ & $\ldots$ & $\cdots$ & $\bigcirc$ & $\cdots$ & $\cdots$ & $\ldots$ & $\bigcirc$ & $\bigcirc$ & & 0 & $\ldots$ & $\ldots$ & $\ldots$ & $\cdots$ \\
\hline $\mathrm{S} 21$ & $\bigcirc$ & $\bigcirc$ & $\bigcirc$ & $\ldots$ & $\ldots$ & $\bigcirc$ & $\ldots$ & $\ldots$ & $\bigcirc$ & $\ldots$ & $\ldots$ & & $\ldots$ & $\ldots$ & $\bigcirc$ & $\ldots$ & $\ldots$ \\
\hline S22 & $\ldots$ & $\ldots$ & $\ldots$ & $\ldots$ & $\ldots$ & $\ldots$ & $\ldots$ & $\ldots$ & $\ldots$ & $\ldots$ & $\ldots$ & & $\ldots$ & $\ldots$ & $\ldots$ & $\ldots$ & $\ldots$ \\
\hline $\mathrm{S} 23$ & $\bigcirc$ & $\bigcirc$ & $\bigcirc$ & $\ldots$ & $\cdots$ & $\bigcirc$ & $\bigcirc$ & $\bigcirc$ & $\cdots$ & $\bigcirc$ & $\bigcirc$ & & $\bigcirc$ & $\bigcirc$ & $\ldots$ & $\ldots$ & $\cdots$ \\
\hline S24 & $\bigcirc$ & $\bigcirc$ & $\bigcirc$ & $\cdots$ & $\cdots$ & 0 & $\bigcirc$ & $\bigcirc$ & $\cdots$ & $\bigcirc$ & $\bigcirc$ & & $\bigcirc$ & $\bigcirc$ & $\cdots$ & $\cdots$ & $\cdots$ \\
\hline $\mathrm{S} 25$ & $\ldots$ & $\ldots$ & $\ldots$ & $\ldots$ & $\cdots$ & $\bigcirc$ & $\cdots$ & $\ldots$ & $\ldots$ & $\cdots$ & $\ldots$ & $\cdots$ & $\ldots$ & $\cdots$ & $\ldots$ & $\ldots$ & $\ldots$ \\
\hline S13 & $\ldots$ & $\ldots$ & $\ldots$ & $\ldots$ & $\cdots$ & $\cdots$ & $\cdots$ & $\ldots$ & $\ldots$ & $\ldots$ & $\ldots$ & $\bigcirc$ & $\ldots$ & $\ldots$ & $\ldots$ & $\ldots$ & $\cdots$ \\
\hline S14 & $\ldots$ & $\ldots$ & $\ldots$ & $\ldots$ & $\ldots$ & $\ldots$ & $\ldots$ & $\ldots$ & $\ldots$ & $\ldots$ & $\ldots$ & $\bigcirc$ & $\ldots$ & $\ldots$ & $\ldots$ & $\ldots$ & $\ldots$ \\
\hline S15 & $\ldots$ & $\ldots$ & $\ldots$ & $\ldots$ & $\ldots$ & $\bigcirc$ & $\ldots$ & $\ldots$ & $\ldots$ & $\ldots$ & $\ldots$ & 0 & $\ldots$ & $\ldots$ & $\ldots$ & $\ldots$ & $\ldots$ \\
\hline $\mathrm{L}^{\mathrm{b}}$ & $\ldots$ & $\ldots$ & $\ldots$ & $\ldots$ & $\ldots$ & $\ldots$ & $\ldots$ & $\ldots$ & $\ldots$ & $\ldots$ & $\ldots$ & $\ldots$ & $\ldots$ & $\ldots$ & $\bigcirc$ & $\ldots$ & $\ldots$ \\
\hline
\end{tabular}

Notes.

a The open circles show the model we simulate.

b The letter "L" means the large inner bar models.

below). $a_{\mathrm{IB}}$ may be less than $600 \mathrm{pc}$. Since the curve $\Omega-\kappa / 2$ in $R<500 \mathrm{pc}$ is rather uncertain, we assume two cases of $a_{\mathrm{IB}}$. Hereafter, we call the inner bar models with $a_{\mathrm{IB}}=200 \mathrm{pc}$ small inner bars and with $a_{\mathrm{IB}}=600$ pc large inner bars.

We assume that the mass of the inner bar (see Section 2.3) is a part of the mass distribution of the nuclear bulge of Launhardt et al. (2002). We give the mass of the inner bar models in Tables 1 and 2. As shown in Figure 1, the mass of the inner bar models are quite smaller than the total mass within the radius of the semimajor axis of the inner bars.

We assume that pattern speeds of the inner bar models are near the local maximum of $\Omega-\kappa / 2$, which is located at about $150 \mathrm{pc}$ (see Figure 2). This is consistent with $N$-body simulations of the formation of nested barred galaxies (Friedli \& Martinet 1993; Rautiainen et al. 2002). We also assume that the inner bars are prograde. In some small inner bar models, we change the pattern speed around the local maximum of $\Omega-\kappa / 2$ to investigate the effect of the pattern speed on the mass inflow rate to the galactic center. The range of the pattern speed in each model is $175-375 \mathrm{~km} \mathrm{~s}^{-1} \mathrm{kpc}^{-1}$. We give the pattern speeds in Table 3.

We use $Q_{T} \equiv\left(F_{\phi} / F_{r}\right)_{\max }$ as a measure of the strength of the inner bar, where $\left(F_{\phi} / F_{r}\right)_{\max }$ is a maximum value of a ratio of the azimuthal component of the gravitational force of the inner bar to the radial component of the total gravitational force within 500 pc. $Q_{T}$ of each model are given in Tables 1 and 2 .

Hereafter, we specify the inner bar models by the name in Tables 1 and 2 and by the value of the pattern speed as S33 $\left(\Omega_{\mathrm{IB}}=300 \mathrm{~km} \mathrm{~s}^{-1} \mathrm{kpc}^{-1}\right)$. In the case of the large inner bar models, we omit the value of the pattern speed, since we assume the same pattern speed for them.

\subsection{Numerical Method}

We use the advection upstream splitting method (AUSM) for numerical hydrodynamics (Liou \& Steffen 1993). The AUSM is one of the flux vector splitting schemes. In the AUSM, advection and propagation of acoustic wave are recognized as physically distinct processes. Therefore, the advective terms and the pressure terms in the flux vector are split separately. This makes the formula of the flux vector at the cell face very simple and leads to a reduction of numerical operations without loss of accuracy. The robustness and good performance of the AUSM in the application to galactic gas simulations are well tested by many authors (Colina \& Wada 2000; Wada \& Norman 2001; Mori et al. 2002). To obtain higher order spatial resolution, we use the second-order MUSCL interpolation with the van Albada limiter function (e.g., Radespiel \& Kroll 1995). The AUSM with the MUSCL interpolation is easy to implement due to its simple form and well suited to capture shock waves in even rarefied medium. In our simulations, we do not use "gas recycling law" (e.g., Athanassoula 1992; Englmaier \& Shlosman 2000). Since our simulation time is much shorter than an exhaustion timescale of gas by star formation, "gas recycling" is not important in our simulation.

In order to resolve gas motion in the Galactic center region, we use two-dimensional polar grids extending from $5 \mathrm{pc}$ to $10 \mathrm{kpc}$ in the Galactic radius. We divided radial grids into 370 logarithmically and azimuthal grids into 300 equally keeping the shape of each cell nearly square. The radial spacing $\Delta R$ of the grids decreases inward. A very high spacial resolution is achieved in the central region, e.g., $\Delta R \approx 0.1 \mathrm{pc}$ at $R=5 \mathrm{pc}$.

We assume isothermal, non-self-gravitating, and nonviscous gas for simplicity. We do not consider a viscous term in the hydrodynamical equations. We use the equation of state of ideal gas with a temperature of $10,000 \mathrm{~K}$, which corresponds to the sound speed of $c_{s} \approx 10 \mathrm{~km} \mathrm{~s}^{-1}$ and random motion of interstellar gas implicitly. We do not consider the star formation and feedback process, such as supernovae and stellar mass loss, in this paper.

We assume a rotationally supported gas disk for the initial state. This disk is flat and has infinitesimal thickness. Its outer radius and mass are $10 \mathrm{kpc}$ and $10^{10} M_{\odot}$, respectively. The initial surface density of the disk is uniform in all models.

The radial outer and inner boundary conditions are free and the azimuthal boundary condition is periodic. We record 


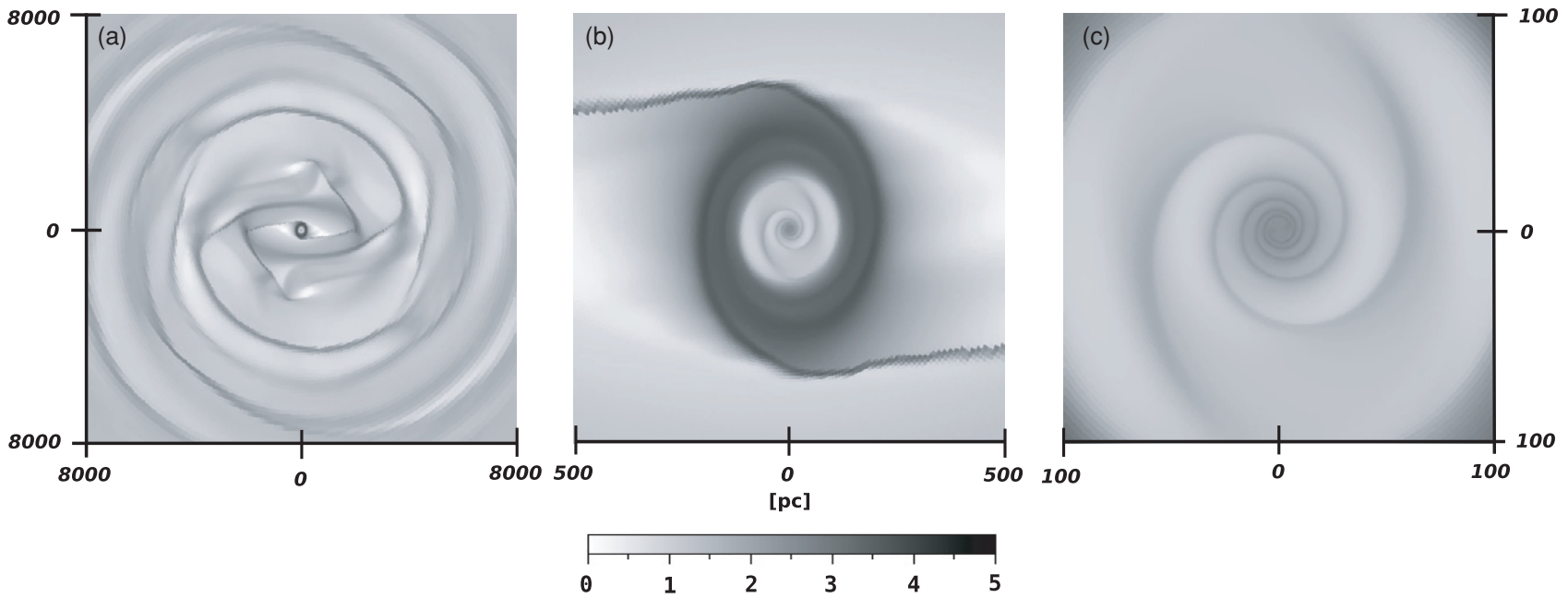

Figure 3. Surface density of gas in the model $\mathrm{N}$ at $t=500 \mathrm{Myr}$. The gray-scale bar shows the surface density of the range of $1-10^{5} M_{\odot} \mathrm{pc}^{-2}$ in the logarithmic scale. The outer bar lies in the horizontal direction in all the panels.

mass flux passing through the inner and the outer boundary for checking mass conservation.

In order to avoid spurious phenomena, we introduce the nonaxisymmetric components, such as the inner bar, the outer bar, and the spiral arms, of the gravitational potential slowly, compared to the rotational speed of each component. We gradually deform the gravitational potential of the inner bar from a spherical shape,

$$
\rho(x, y, z)=\rho_{0}^{\prime}\left(1-\frac{x^{2}+y^{2}+z^{2}}{r_{0}^{2}}\right),
$$

where $\rho_{0}^{\prime}=\frac{15 M_{\mathrm{IB}}}{8 \pi r_{0}^{2}}$ and $r_{0}=\frac{a+b}{2}$, to its assumed one from $t=100$ to $250 \mathrm{Myr}$ as in Athanassoula (1992). We also similarly introduce the Fourier component of the gravitational potential of the outer bar and the spiral arms given by Bissantz et al. (2003) from $t=0$ to $100 \mathrm{Myr}$.

We use the super computer SR11000/K1 of the Hokkaido University Information Initiative Center (IIC) for our simulations.

\section{NUMERICAL RESULTS}

We perform the hydrodynamical simulations for various masses and axial ratios of the inner bar systematically. In the small inner bar models, we also vary their pattern speed.

We find a large amount of gas concentration to the Galactic center in both sizes of the inner bar models. In small inner bar models, the inner bars induce gas inflow to the Galactic center for $0.05<Q_{T}<0.3$ without destroying the $200 \mathrm{pc}$ gas ring, if $\Omega_{\mathrm{IB}} \approx(\Omega-\kappa / 2)_{\max } \approx 300 \mathrm{~km} \mathrm{~s}^{-1} \mathrm{kpc}^{-1}$ for $0.12 \gtrsim Q_{T} \gtrsim 0.3$ and if $\Omega_{\mathrm{IB}} \approx 225 \mathrm{~km} \mathrm{~s}^{-1} \mathrm{kpc}^{-1}$ for $Q_{T} \gtrsim 0.05$. On the other hand, in large inner bar models, gas concentration occurs if $Q_{T}>0.1$ and the inner bar destroys the $200 \mathrm{pc}$ gas ring. In the following subsections, we describe the results in more detail.

\subsection{The No-Inner Bar Case}

We perform hydrodynamical simulation in the Galaxy model without the inner bar to compare with the models with the inner bars. We show result of the no-inner bar model (model $\mathrm{N}$ ) in Figure 3.
Gas ridges are formed in the outer bar region by $t=100 \mathrm{Myr}$. Gas in the Galactic disk flows into the central region $(R<$ $300 \mathrm{pc}$ ) along the gas ridges. This result is almost the same results of Bissantz et al. (2003). In our numerical results, a gas ring is formed at the radius of $150-250 \mathrm{pc}$. Its mass is almost constant at the value of $\approx 3 \times 10^{8} M_{\odot}$ after $t=100 \mathrm{Myr}$. The mass and size of the ring correspond to the $\mathrm{CMZ}$, of which extent is $-1.5 \leqslant l \leqslant 2^{\circ}$ and mass is $(5-10) \times 10^{7} M_{\odot}$. We find similar gas rings in the models with inner bars. Hereafter we call these rings the $200 \mathrm{pc}$ gas rings.

The radius of the $200 \mathrm{pc}$ gas ring is well inside the position of the inner Lindblad resonance (ILR) of the outer bar $(\sim 750 \mathrm{pc})$. This result agrees with Regan \& Teuben (2003). They showed that the size of the nuclear ring is related to the population of $x_{2}$ orbits, rather than the position of ILRs of an outer bar when gas motion is in the nonlinear regime of hydrodynamics in the barred potential.

Inside the 200 pc gas ring, there are weak gas spirals. Their pattern speed agrees with the pattern speed of the outer bar. These spirals are density waves found by Englmaier \& Shlosman (2000). Englmaier \& Shlosman (2000) show that gaseous spirals are formed inside the ILR of a bar in their numerical simulations of non-self-gravitating gaseous disks and that such gaseous spirals are supported by pressure force and stationary in the bar frame. The gaseous spirals in our simulation have a similar property. Hereafter we call these spirals the nuclear spirals. The nuclear spirals become more tightly wound as approaching the Galactic center. Near 20 pc from the center, nuclear spirals have highly tight winding. An average mass inflow rate from 100 to 500 Myr is very small, $\approx 3.6 \times 10^{-4} M_{\odot} \mathrm{yr}^{-1}$. This radial gas inflow may be due to the nuclear spirals, since the total gravitational torque on the gas in the nuclear spirals region is consistent with the average mass inflow rate. In order to confirm this, we calculate the total gravitational torque on the gas inside $R=60 \mathrm{pc}$ from the outer bar. The time-averaged total gravitational torque within $R=60$ pc between 100 and $500 \mathrm{Myr}$ is $-7.6 \times 10^{49} \mathrm{~g} \mathrm{~cm}^{2} \mathrm{~s}^{-2}$. The mass inflow rate is as large as $\sim 10^{-3} M_{\odot} \mathrm{yr}^{-1}$ by this torque. This is consistent with the average mass inflow rate from 100 to $500 \mathrm{Myr}$.

Such nuclear spirals were not formed in the simulations of Bissantz et al. (2003). This may be due to the lack of the spacial resolution in the nuclear spirals region in the simulations of 


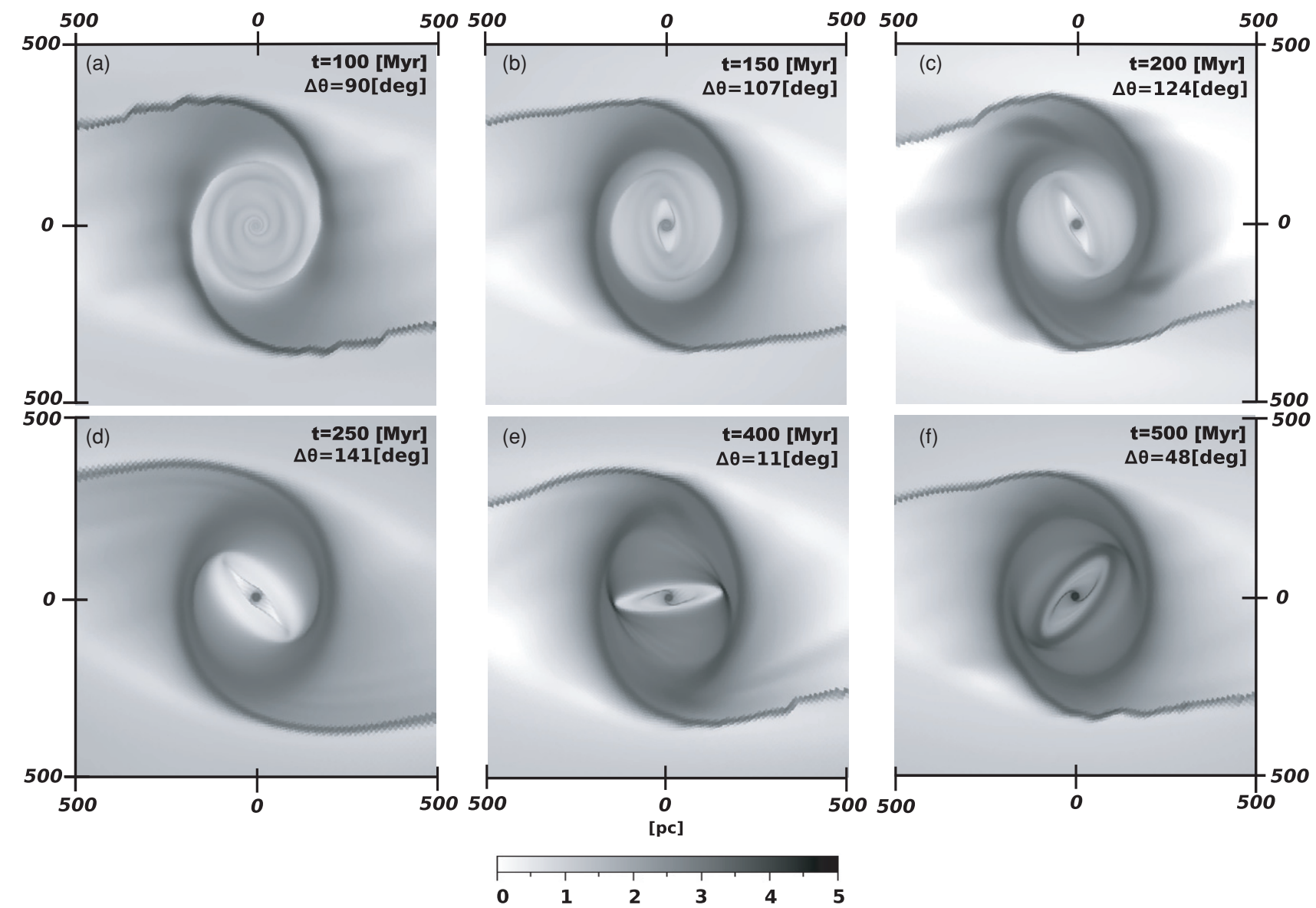

Figure 4. Time variation of the surface density of the central $\mathrm{kpc}$ of the model $\mathrm{S} 33\left(300 \mathrm{~km} \mathrm{~s}^{-1} \mathrm{kpc}^{-1}\right)$. The gray-scale range is the same as in Figure 3 . We show the calculation time and the angle between the outer bar and the inner bar, $\Delta \theta$, in the upper right corner in each panel.

Bissantz et al. (2003). Englmaier \& Shlosman (2000) have shown that in simulations with insufficient spacial resolution to resolve nuclear spiral waves, they are quickly damped out due to numerical viscosity.

\subsection{The Small Inner Bar Models}

We find that a large amount of gas concentrates to the Galactic center in the small inner bar models with $Q_{T} \gtrsim 0.05$ in some range of $\Omega_{\mathrm{IB}}$. We divide our results into two cases, the high gas mass concentration case and the low gas mass concentration case.

If $Q_{T} \gtrsim 0.12$ (S42, S43, S33, and S34), a high gas mass concentration to the Galactic center occurs for both $\Omega_{\mathrm{IB}} \approx$ $(\Omega-\kappa / 2)_{\max } \approx 300 \mathrm{~km} \mathrm{~s}^{-1} \mathrm{kpc}^{-1}$ and $\Omega_{\mathrm{IB}} \sim 225 \mathrm{~km} \mathrm{~s}^{-1} \mathrm{kpc}^{-1}$. If $0.05 \lesssim Q_{T} \lesssim 0.12$ (S41, S32, and S23), the high gas mass concentration to the Galactic center occurs only for $\Omega_{\mathrm{IB}} \sim 225 \mathrm{~km} \mathrm{~s}^{-1} \mathrm{kpc}^{-1}$. One exception is the model S24, in which the high gas mass concentration occurs for both $\Omega_{\mathrm{IB}} \approx(\Omega-\kappa / 2)_{\max }$ and $\Omega_{\mathrm{IB}} \sim 225 \mathrm{~km} \mathrm{~s}^{-1} \mathrm{kpc}^{-1}$ in spite of $0.05 \lesssim Q_{T} \lesssim 0.12$.

\subsubsection{The High Gas Mass Concentration Cases}

Here, we describe the results of the model S33 $\left(\Omega_{\mathrm{IB}}=\right.$ $300 \mathrm{~km} \mathrm{~s}^{-1} \mathrm{kpc}^{-1}$ ) in detail, since the time evolution of gas distribution in the inner bar region is similar to the high gas mass concentration cases.

We show the time evolution of the surface density of gas in the central $1 \mathrm{kpc}$ square in the model $\mathrm{S} 33\left(\Omega_{\mathrm{IB}}=300 \mathrm{~km} \mathrm{~s}^{-1} \mathrm{kpc}^{-1}\right)$ in Figure 4. One of the characteristic gas distributions is straight shocks inside the inner bar (see Figure 5). These shocks appear after the inner bar potential is introduced and become stronger with the calculation time (see Figures $4 \mathrm{~d}-\mathrm{f}$ ). These shocks extend from the Galactic central disk to the inner edge of the $200 \mathrm{pc}$ gas ring and are efficient to supply a large amount of gas to the Galactic center. A massive nuclear gas disk forms in $R \lesssim 15 \mathrm{pc}$. Its mass reaches as large as $10^{7} M_{\odot}$ at $t=500 \mathrm{Myr}$. Hereafter we call this disk the nuclear gas disk.

An elliptical gas ring is formed around the inner bar and is elongated along the inner bar (see Figure 4f). A similar elliptical gas ring is shown in Maciejewski et al. (2002). The shape and surface density of this ring changes as the inner bar rotates. In Figure 5, the ellipticity of the ring is larger at $\Delta \theta=90^{\circ}$ than at $\Delta \theta=0^{\circ}$, while the surface density of the ring is higher at $\Delta \theta=0^{\circ}$ than at $\Delta \theta=90^{\circ}$, where $\Delta \theta$ is the angle between major axes of the inner bar and the outer bar. The velocity fields in the elliptical ring are smoothly connected to that of the surrounding gas.

In Figure 6 we show the time evolution of the gas mass within $20 \mathrm{pc}, M_{20}(t)$, in the model S33 $\left(\Omega_{\mathrm{IB}}=300 \mathrm{~km} \mathrm{~s}^{-1} \mathrm{kpc}^{-1}\right)$. As the deformation of the inner bar proceeds $(t=100-250 \mathrm{Myr})$, $M_{20}(t)$ rapidly increases with time. Then, $M_{20}(t)$ saturates $(t=250-350 \mathrm{Myr})$. A similar phenomenon was reported by Maciejewski et al. (2002). Maciejewski et al. (2002) showed that an inner bar keeps the gas away from the Galactic center and the gas inflow due to the inner bar is negligible after it reaches its full strength. In the corresponding stage, in our simulations, velocity fields and gas distribution inside the $200 \mathrm{pc}$ 

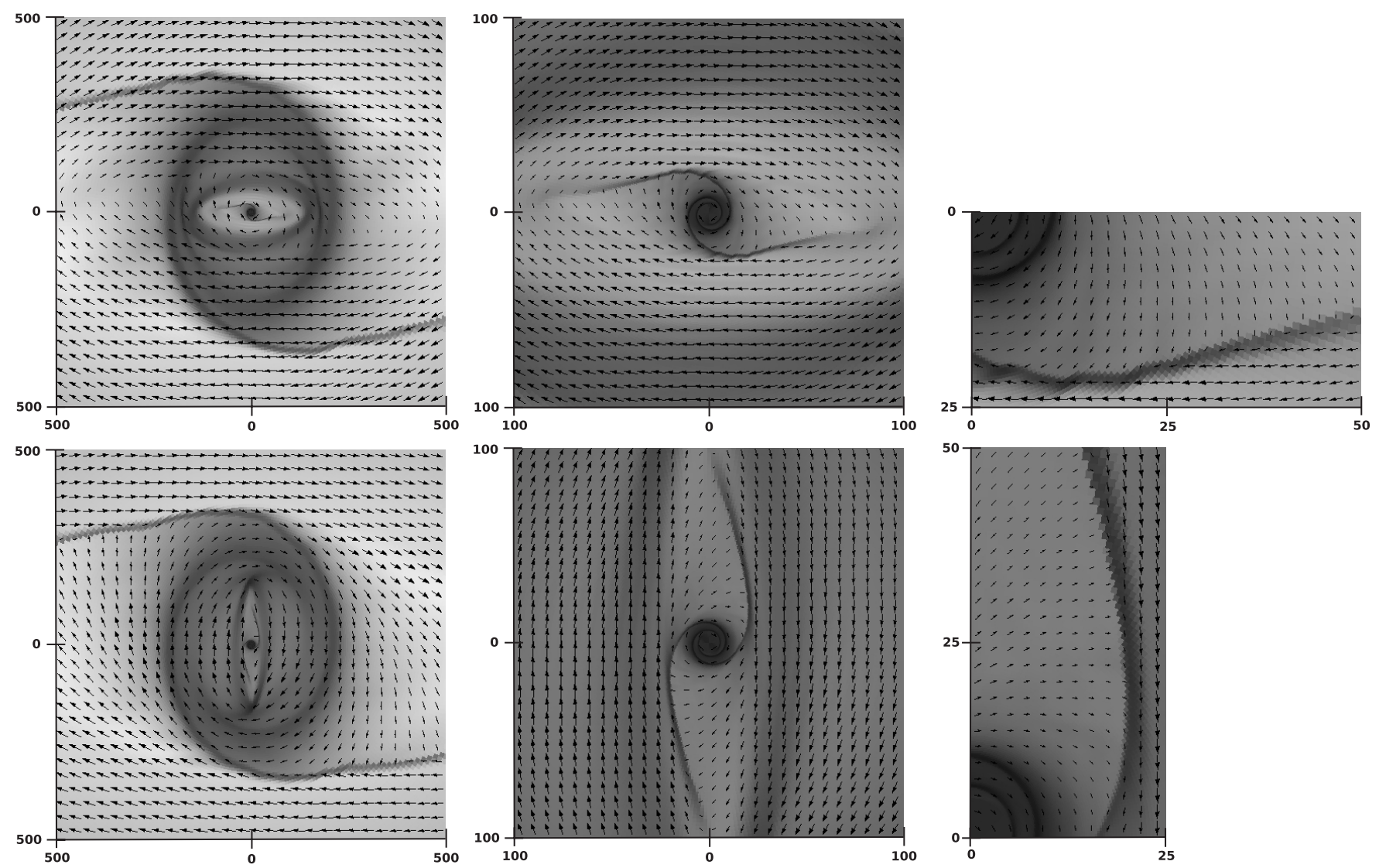

Figure 5. Velocity fields of the model S33 $\left(300 \mathrm{~km} \mathrm{~s}^{-1} \mathrm{kpc}^{-1}\right)$. The gray-scale range is the same as in Figure 4. Upper panels: $\Delta \theta=0^{\circ}(t=490 \mathrm{Myr})$. Lower panels: $\Delta \theta=90^{\circ}(t=497 \mathrm{Myr})$. Note that the arrows show velocities in these regions in relative scale.

gas ring is perturbed by the inner bar. After velocity fields and gas distribution is quasi-steady, the increase of gas inflow to the Galactic center begins at $t=350 \mathrm{Myr}$. This second inflow continues to the end of the simulations. $M_{20}(t)$ attains $\sim 10^{7} M_{\odot}$ at $t=500 \mathrm{Myr}$. An average second mass inflow rate is $\sim 10^{7} M_{\odot} / 100 \mathrm{Myr} \approx 0.1 M_{\odot} \mathrm{yr}^{-1}$. We discuss the difference between our results and that of Maciejewski et al. (2002) in Section 4.

Occurrence of the second mass inflow depends on the pattern speed of the inner bar. We show the time evolution of $M_{20}(t)$ for various pattern speeds of the inner bar in the model S33 in the lower panel of Figure 6. Figure 6 shows that the second mass inflow occurs when the pattern speed is $290-325 \mathrm{~km} \mathrm{~s}^{-1} \mathrm{kpc}^{-1}$ and $200-225 \mathrm{~km} \mathrm{~s}^{-1} \mathrm{kpc}^{-1}$ in the model S33. We summarize $M_{20}(t=500 \mathrm{Myr})$ for small inner bar models in Table 4. In this table, we denote models in which the second mass inflow occurs by bold letters. We note the models by daggers, in which $M_{20}(t=500 \mathrm{Myr})$ exceeds the stellar mass within $20 \mathrm{pc}$ $M_{\star}(<20 \mathrm{pc}) \approx 2 \times 10^{7} M_{\odot}$ (see Figure 14 in Launhardt et al. 2002). In this case, we should solve self-consistently both the motion of gas and stars in the Galactic central region. Double daggers show the models in which the second mass inflows begin just before the end of the simulation. In these models, more mass will inflow into the Galactic center, if we continue the simulations.

Mass of the nuclear gas disk increases with time periodically by the second mass inflow. Similar periodicity have been reported in Shlosman \& Heller (2002a). This case may be closely related with resonance phenomena between the outer bar and the inner bar. In our results, sufficient elongation for a small inner bar and a suitable $\Omega_{\text {IB }}$ are needed for the second mass inflow.

\subsubsection{The Low Gas Mass Concentration Cases}

In small inner bar models with $Q_{T}<0.05$, the gas mass concentration to the Galactic center is small (S31, S21, S22, S13, and S14). In these models, two loose gas spirals appear in the inner bar region instead of straight shocks. These nuclear gas spirals become tightly wound near the center and a less massive gas disk appears in $R<20 \mathrm{pc}$ from the center.

The low gas mass concentration is due to the absence of the second mass inflow. We show the time evolution of $M_{20}(t)$ in the model S21 $\left(\Omega_{\mathrm{IB}}=300 \mathrm{~km} \mathrm{~s}^{-1} \mathrm{kpc}^{-1}\right)$ by a dashed line in the upper left panel of Figure 6. This figure shows that $M_{20}(t)$ saturates after the first mass increase and the second mass inflow does not occur till the end of the simulation. We test the time evolution of $M_{20}(t)$ of the model S21 for various pattern speeds, as shown in the upper right panel of Figure 6. There is no second mass inflow in a range of $\Omega_{\mathrm{IB}}=175-325 \mathrm{~km} \mathrm{~s}^{-1} \mathrm{kpc}^{-1}$. Thus, we conclude that the second mass inflow needs $Q_{T} \gtrsim 0.05$.

We address the characteristic gas distribution seen in the low gas mass concentration models, since it is clear evidence of an inner weak bar. In Figure 7, we show the snapshots of the surface density of the model S2 1 for two pattern speeds, $\Omega_{\mathrm{IB}}=200$ and $300 \mathrm{~km} \mathrm{~s}^{-1} \mathrm{kpc}^{-1}$. In both models, the two loose gas spirals are formed in the inner bar region and are surrounded by the gas rings. A similar structure is observed in the double-barred galaxy, NGC 1097. In this galaxy, the loose gas spirals are 

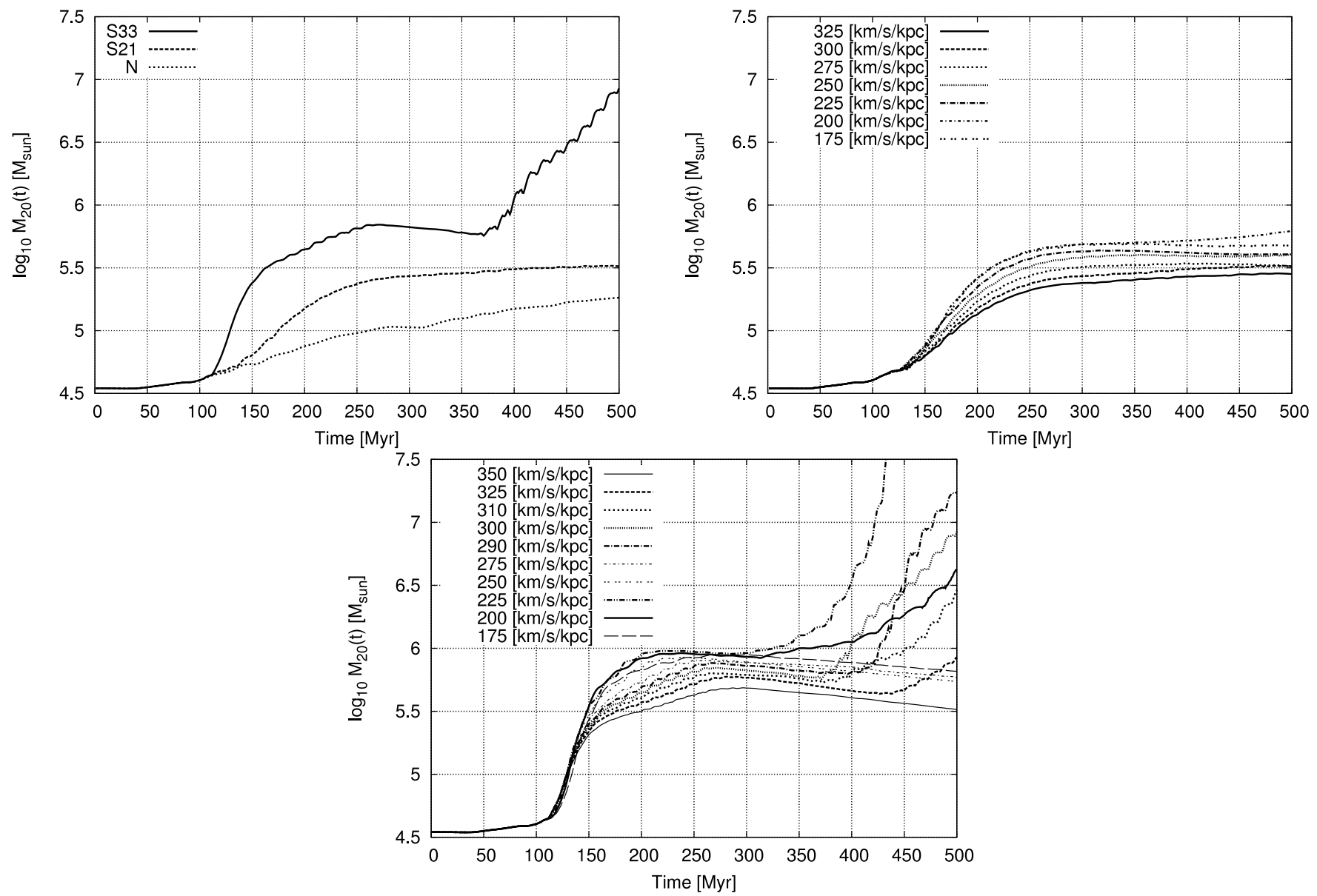

Figure 6. $M_{20}(t)$ of three different models: S33 $\left(\Omega_{\mathrm{IB}}=300 \mathrm{~km} \mathrm{~s}^{-1} \mathrm{kpc}^{-1}\right), \mathrm{S} 21\left(\Omega_{\mathrm{IB}}=300 \mathrm{~km} \mathrm{~s}^{-1} \mathrm{kpc}^{-1}\right.$ ), and N (upper left panel). $M_{20}(t)$ of the model S21 (upper right panel) and the model S33 (lower panel) for various pattern speeds of the inner bar. The horizontal and vertical axes are the calculation time and logarithmic value of $M_{20}(t) / M_{\odot}$, respectively.
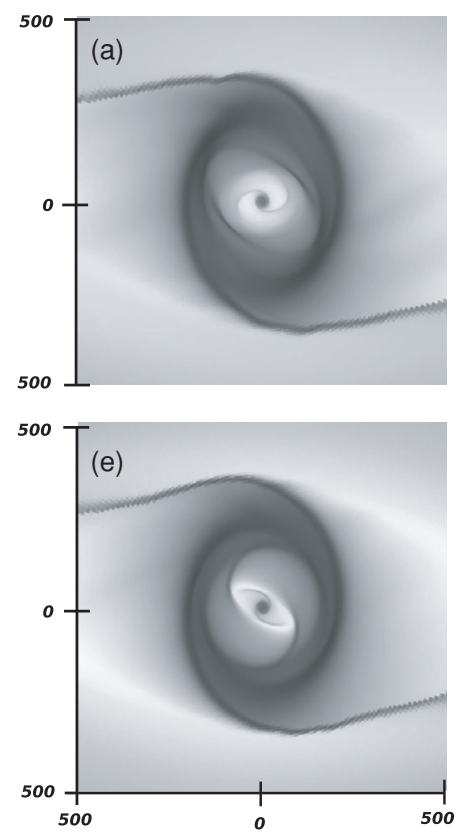
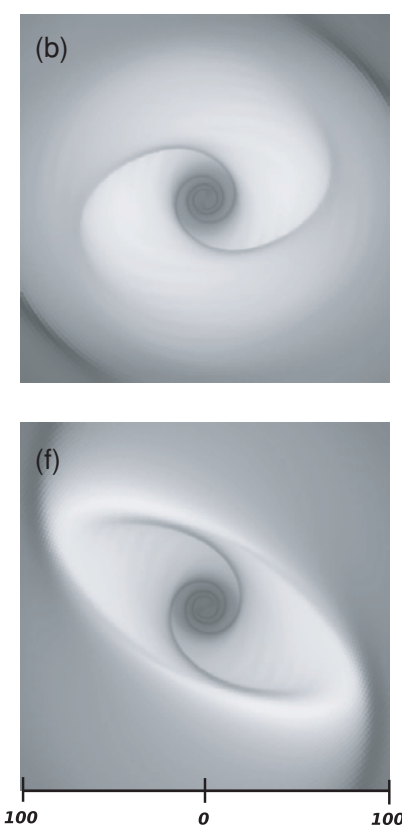
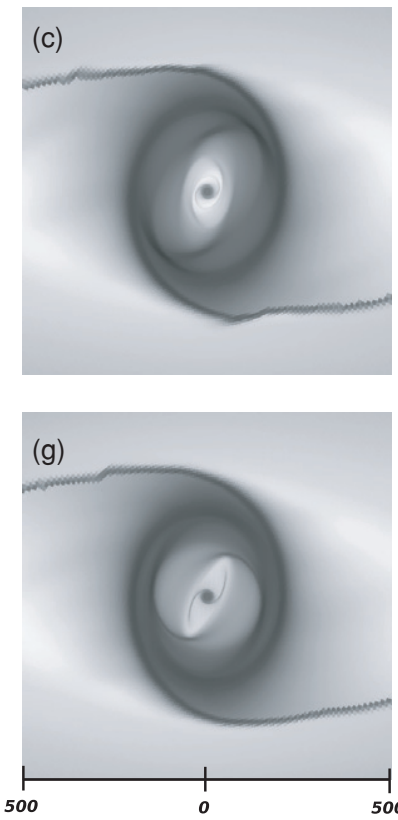

500100
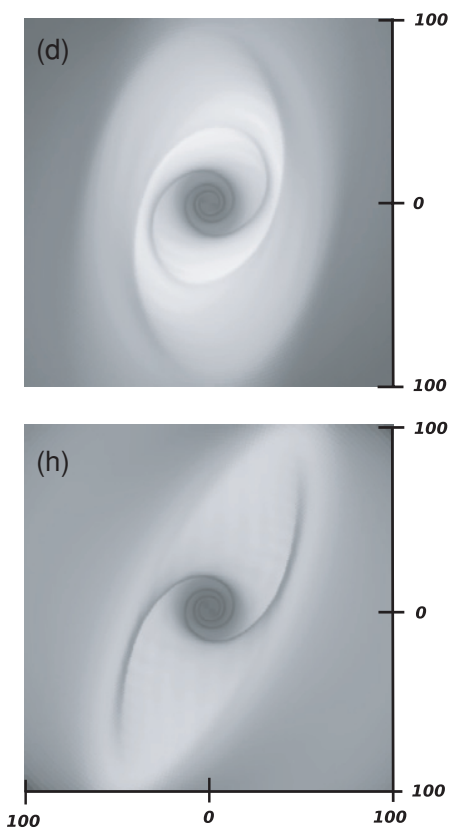

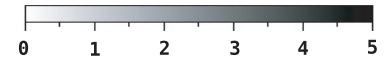

Figure 7. Surface density distribution of the model S21. Upper row: the model S21 $\left(\Omega_{\mathrm{IB}}=300 \mathrm{~km} \mathrm{~s}^{-1} \mathrm{kpc}^{-1}\right)$. Lower row: the model S21 $\left(\Omega_{\mathrm{IB}}=200 \mathrm{~km} \mathrm{~s}^{-1} \mathrm{kpc}^{-1}\right)$. First and second columns: $\Delta \theta=0^{\circ}$. Third and fourth columns: $\Delta \theta=90^{\circ}$. 
Table 4

High Gas Mass Concentration Cases in the Small Inner Bar Models

\begin{tabular}{|c|c|c|c|c|c|c|c|c|c|c|c|c|c|c|c|c|c|}
\hline \multirow[t]{2}{*}{ Model Name } & \multicolumn{17}{|c|}{ Pattern Speed of the Inner Bar $\Omega_{\mathrm{IB}}\left(\mathrm{km} \mathrm{s}^{-1} \mathrm{kpc}^{-1}\right)$} \\
\hline & 175 & 200 & 225 & 230 & 240 & 250 & 260 & 270 & 275 & 280 & 290 & 300 & 310 & 320 & 325 & 350 & 375 \\
\hline S41 & $\ldots$ & $2.0 \mathrm{E}+6^{\mathrm{a}}$ & $\ldots$ & $8.1 \mathrm{E}+6^{\mathrm{a}}$ & $8.7 E+6^{a}$ & $1.8 \mathrm{E}+6^{\mathrm{a}}$ & $7.6 \mathrm{E}+5$ & $7.0 \mathrm{E}+5$ & $\ldots$ & $5.9 \mathrm{E}+5$ & $6.3 \mathrm{E}+5$ & $5.6 \mathrm{E}+5$ & $4.7 \mathrm{E}+5$ & $4.4 \mathrm{E}+5$ & $\ldots$ & $\ldots$ & $\ldots$ \\
\hline S42 & $6.7 \mathrm{E}+5$ & $3.2 \mathrm{E}+6^{\mathrm{a}}$ & $1.5 \mathrm{E}+8^{\mathrm{b}}$ & $\ldots$ & $\ldots$ & $6.7 \mathrm{E}+5$ & $\ldots$ & $\ldots$ & $6.5 \mathrm{E}+5^{\mathrm{a}, \mathrm{c}}$ & $\ldots$ & $9.6 \mathrm{E}+6^{\mathrm{a}}$ & $1.5 \mathrm{E}+7^{\mathrm{a}}$ & $7.5 \mathrm{E}+5^{\mathrm{a}, \mathrm{c}}$ & $\ldots$ & $1.6 \mathrm{E}+6^{\mathrm{a}}$ & $5.7 \mathbf{E}+5^{\mathrm{a}, \mathrm{c}}$ & $3.5 \mathrm{E}+5$ \\
\hline $\mathrm{S} 43$ & $4.7 \mathrm{E}+5$ & $2.0 \mathrm{E}+7^{\mathrm{a}, \mathrm{b}}$ & $7.8 \mathrm{E}+7^{\mathrm{a}, \mathrm{b}}$ & $\ldots$ & $\ldots$ & $3.8 \mathrm{E}+5$ & $\ldots$ & $\ldots$ & $2.1 \mathrm{E}+6^{\mathrm{a}}$ & $\ldots$ & $1.1 \mathrm{E}+\boldsymbol{8}^{\mathrm{a}, \mathrm{b}}$ & $7.7 \mathbf{E}+7^{\mathrm{a}, \mathrm{b}}$ & $4.8 \mathrm{E}+7^{\mathrm{a}, \mathrm{b}}$ & $\ldots$ & $2.4 \mathrm{E}+7^{\mathrm{a}, \mathrm{b}}$ & $9.5 \mathrm{E}+6^{\mathrm{a}}$ & $4.5 \mathrm{E}+6^{\mathrm{a}}$ \\
\hline S31 & $\ldots$ & $\ldots$ & $\ldots$ & $\ldots$ & $\ldots$ & $\ldots$ & $\ldots$ & $\ldots$ & $\ldots$ & $\ldots$ & $\ldots$ & $3.4 \mathrm{E}+5$ & $\ldots$ & $\ldots$ & $\ldots$ & $\ldots$ & $\ldots$ \\
\hline S32 & $6.0 \mathrm{E}+5$ & $2.2 \mathrm{E}+6^{\mathrm{a}}$ & $1.5 \mathrm{E}+7^{\mathrm{a}}$ & $\ldots$ & $\ldots$ & $5.7 \mathrm{E}+5$ & $5.0 \mathrm{E}+5$ & $4.9 \mathrm{E}+5$ & $\ldots$ & $4.8 \mathrm{E}+5$ & $4.4 \mathrm{E}+5$ & $4.1 \mathrm{E}+5$ & $4.0 \mathrm{E}+5$ & $3.5 \mathrm{E}+5$ & $\ldots$ & $\ldots$ & $\ldots$ \\
\hline S33 & $6.6 \mathrm{E}+5$ & $4.2 \mathrm{E}+6^{\mathrm{a}}$ & $1.4 \mathrm{E}+8^{\mathrm{a}, \mathrm{b}}$ & $\ldots$ & $\ldots$ & $5.4 \mathrm{E}+5$ & & $\ldots$ & $5.9 \mathrm{E}+5$ & $\ldots$ & $1.7 \mathrm{E}+7^{\mathrm{a}}$ & $8.5 \mathrm{E}+6^{\mathrm{a}}$ & $2.9 \mathrm{E}+6^{\mathrm{a}}$ & $\ldots$ & $8.5 \mathrm{E}+5^{\mathrm{a}, \mathrm{c}}$ & $3.3 \mathrm{E}+5$ & $\ldots$ \\
\hline S34 & $\ldots$ & $\ldots$ & $\ldots$ & $\ldots$ & $\ldots$ & $6.7 \mathrm{E}+5$ & $6.2 \mathrm{E}+5$ & $5.3 \mathrm{E}+5$ & $\ldots$ & $4.2 \mathrm{E}+6$ & $1.6 \mathrm{E}+8^{\mathrm{b}}$ & $8.5 \mathrm{E}+7^{\mathrm{b}}$ & $8.7 E+7^{b}$ & $\ldots$ & $\ldots$ & $\ldots$ & $\ldots$ \\
\hline S35 & $\ldots-$ & $\ldots$ & $\ldots$ & $\ldots$ & $\ldots$ & $1.5 \mathrm{E}+5$ & $\ldots$ & $\ldots$ & $\ldots$ & $2.1 \mathrm{E}+8^{\mathrm{b}}$ & $1.0 \mathrm{E}+8^{\mathrm{b}}$ & $1.2 \mathrm{E}+8^{\mathrm{b}}$ & 4.1E+7 & $\ldots$ & $\ldots$ & $\ldots$ & $\ldots$ \\
\hline S 21 & $4.8 \mathrm{E}+5$ & $6.2 \mathrm{E}+5$ & $4.1 \mathrm{E}+5$ & $\ldots$ & $\cdots$ & $4.0 \mathrm{E}+5$ & $\cdots$ & $\ldots$ & $3.3 \mathrm{E}+5$ & $\ldots$ & $\ldots$ & $3.3 \mathrm{E}+5$ & $\ldots$ & $\cdots$ & $2.8 \mathrm{E}+5$ & $\cdots$ & $\cdots$ \\
\hline S22 & $\ldots$ & $\ldots$ & $\ldots$ & $\ldots$ & $\ldots$ & $\ldots$ & $\ldots$ & $\ldots$ & $\ldots$ & $\ldots$ & $\ldots$ & $3.2 \mathrm{E}+5$ & $\ldots$ & $\ldots$ & $\ldots$ & $\ldots$ & $\ldots$ \\
\hline S23 & $7.4 \mathrm{E}+5$ & $1.4 \mathrm{E}+6^{\mathrm{a}}$ & $3.4 \mathrm{E}+7^{\mathrm{a}, \mathrm{b}}$ & $\ldots$ & $\ldots$ & $3.0 \mathrm{E}+5$ & $3.2 \mathrm{E}+5$ & $3.4 \mathrm{E}+5$ & $\ldots$ & $3.8 \mathrm{E}+5$ & $3.7 \mathrm{E}+5$ & $3.4 \mathrm{E}+5$ & $3.2 \mathrm{E}+5$ & $3.1 \mathrm{E}+5$ & $\ldots$ & $\ldots$ & $\ldots$ \\
\hline S24 & $1.1 \mathrm{E}+6$ & $3.9 \mathrm{E}+6^{\mathrm{a}}$ & $1.3 \mathrm{E}+8^{\mathrm{a}, \mathrm{b}}$ & $\ldots$ & $\ldots$ & $3.6 \mathrm{E}+5$ & $3.9 \mathrm{E}+5$ & 4.1E+5 & $\ldots$ & $1.4 \mathrm{E}+6^{\mathrm{a}}$ & $9.7 \mathrm{E}+5^{\mathrm{a}}$ & $5.1 \mathrm{E}+5$ & $4.4 \mathrm{E}+5$ & $3.7 \mathrm{E}+5$ & $\ldots$ & $\ldots$ & $\ldots$ \\
\hline S25 & $\ldots$ & $\ldots$ & $\ldots$ & $\ldots$ & $\ldots$ & $4.8 \mathrm{E}+5$ & $\ldots$ & $\ldots$ & $\ldots$ & $\ldots$ & $\ldots$ & $\ldots$ & $\ldots$ & $\ldots$ & $\ldots$ & $\ldots$ & $\ldots$ \\
\hline S13 & $\ldots$ & $\ldots$ & $\ldots$ & $\ldots$ & $\ldots$ & $\ldots$ & $\ldots$ & $\ldots$ & $\ldots$ & $\ldots$ & $\ldots$ & $1.7 \mathrm{E}+5$ & $\ldots$ & $\ldots$ & $\ldots$ & $\ldots$ & $\ldots$ \\
\hline S14 & $\cdots$ & $\cdots$ & $\cdots$ & $\cdots$ & $\cdots$ & $\ldots$ & $\cdots$ & $\cdots$ & $\cdots$ & $\cdots$ & $\cdots$ & $2.4 \mathrm{E}+5$ & $\cdots$ & $\cdots$ & $\cdots$ & $\cdots$ & $\cdots$ \\
\hline S15 & $\cdots$ & $\cdots$ & $\ldots$ & $\ldots$ & $\ldots$ & $2.3 \mathrm{E}+5$ & $\ldots$ & $\ldots$ & $\ldots$ & $\ldots$ & $\ldots$ & $2.2 \mathrm{E}+5$ & $\ldots$ & $\cdots$ & $\ldots$ & $\cdots$ & $\ldots$ \\
\hline
\end{tabular}

Notes.

a The models in which $M_{20}(t=500 \mathrm{Myr})>M_{\star}(<20 \mathrm{pc}) \approx 2 \times 10^{7} M_{\odot}$.

${ }^{b}$ The models in which the second mass inflow starts just before the end of the simulation.

The models in which the second mass flow rate changes periodically. 


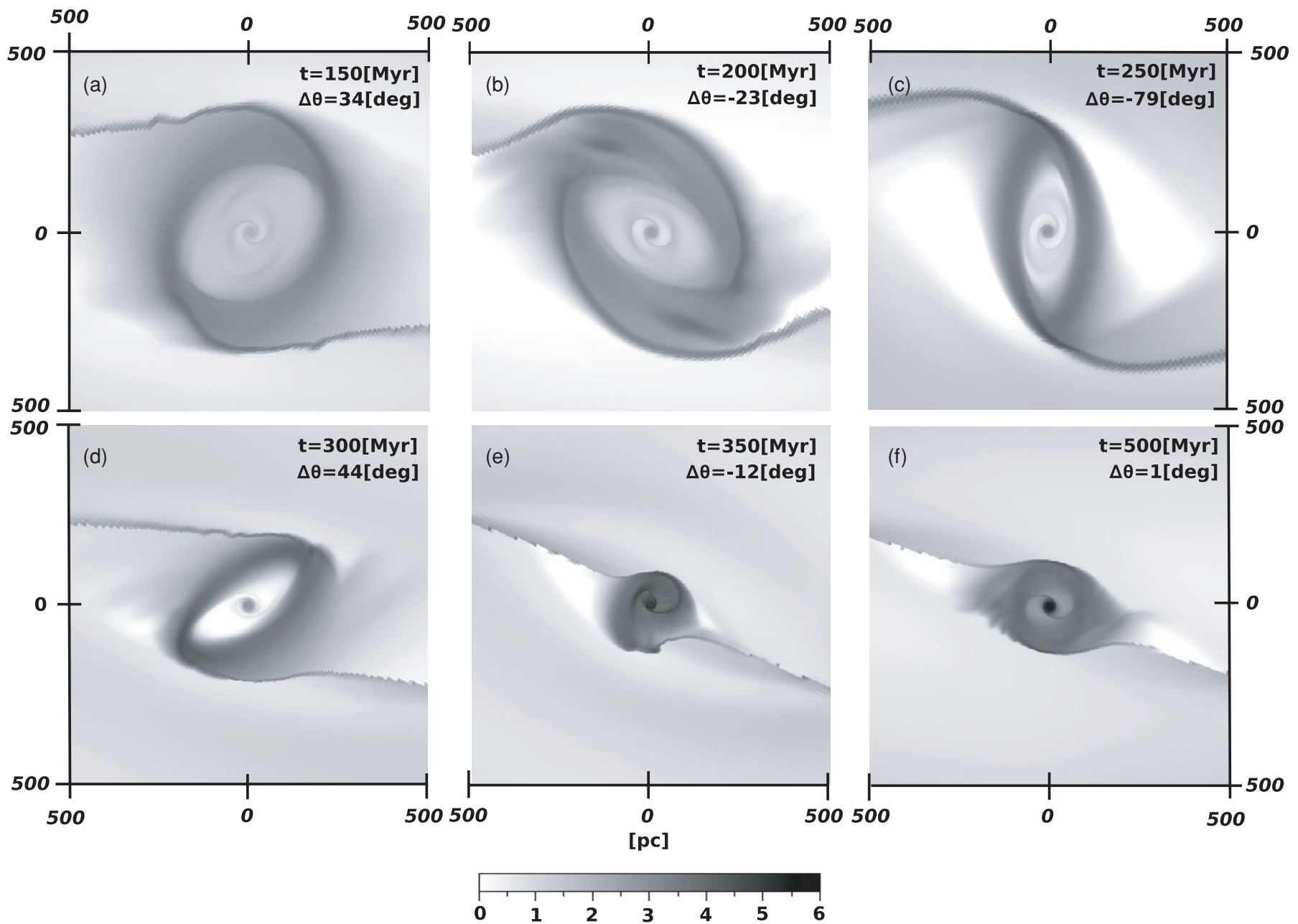

Figure 8. Time variation of the surface density of gas in the model L42. The gray-scale bar shows the surface density of the range $1-10^{6} M_{\odot} \mathrm{pc}^{-2}$ in the logarithmic scale. The values in the upper right corner in each panel are the same as in Figure 4.

observed within the starburst ring (Prieto et al. 2005; Fathi et al. 2006). Contrary to Prieto et al. (2005), it is possible that loose gas spirals are formed by an inner bar without any peculiar assumption.

\subsection{The Large Inner Bar Models}

\subsubsection{The High Gas Mass Concentration Cases}

In large inner bar models with $Q_{T} \gtrsim 0.11$ (L42, L43, L33, $\mathrm{L} 34$, and L35), a large amount of gas concentrates to the Galactic center for $\Omega_{\mathrm{IB}}=325 \mathrm{~km} \mathrm{~s}^{-1} \mathrm{kpc}^{-1}$. In Figure 8 we show the time evolution of the surface density of gas of the model L42. In $t=100-250 \mathrm{Myr}$, elongation of the $200 \mathrm{pc}$ gas ring increases. As can be seen in Figures 8(b)-(d), the 200 pc gas ring is highly elongated in $t=150-300 \mathrm{Myr}$. At $t=350 \mathrm{Myr}$, the $200 \mathrm{pc}$ gas ring shrinks to less than $R=150 \mathrm{pc}$ (see Figure $8 \mathrm{f}$ ). Then, a large part of the gas of the $200 \mathrm{pc}$ gas ring rapidly concentrates into the Galactic center and a very massive gas disk is formed at the center. The mass of the disk highly exceeds $10^{8} M_{\odot}$. The final value of $M_{20}(t)$ is unreal because of the same reason described in Section 3.2.1.

\subsubsection{The Low Gas Mass Concentration Cases}

In the case of $Q_{T}<0.11$ (L41, L31, L32, L22, L23, L24, L13, L14, and L15), a large amount of gas do not concentrate to the Galactic center. The inner bar changes the shape of the $200 \mathrm{pc}$ gas ring into more elliptical. The orientation of the deformed $200 \mathrm{pc}$ gas ring is almost parallel to the inner bar. In these models, there is no enhancement of the mass inflow rate to the center. The average mass inflow rate over the simulation time is as small as the no-inner-bar case.

\section{DISCUSSION}

\subsection{Mass Supply Due to Nested Bars}

We have shown that the mass supply process due to nested bars is a very efficient process by the numerical simulations. There are possible scenarios of the mass supply to the Galactic center. Athanassoula (1992) showed that gas ridges can reach a Galactic center if a large-scale bar is very strong. However, the axial ratio of the outer bar of our Galaxy is $\approx 3$ (Stanek et al. 1997; Rattenbury et al. 2007). Hence, it is unlikely that mass supply to the Galactic center is due to the "past" strong outer bar. Fukuda et al. (2000) simulated self-gravitational instability of a nuclear gas ring and showed that a part of gas in the ring falls into a Galactic center, since the gas transfers its angular momentum to a very massive clump, which is formed due to the fragmentation of the gas ring and subsequent mass accretion by surrounding gas. This process can explain mass supply to the Galactic center if the $\mathrm{CMZ}$ corresponds to such a nuclear 


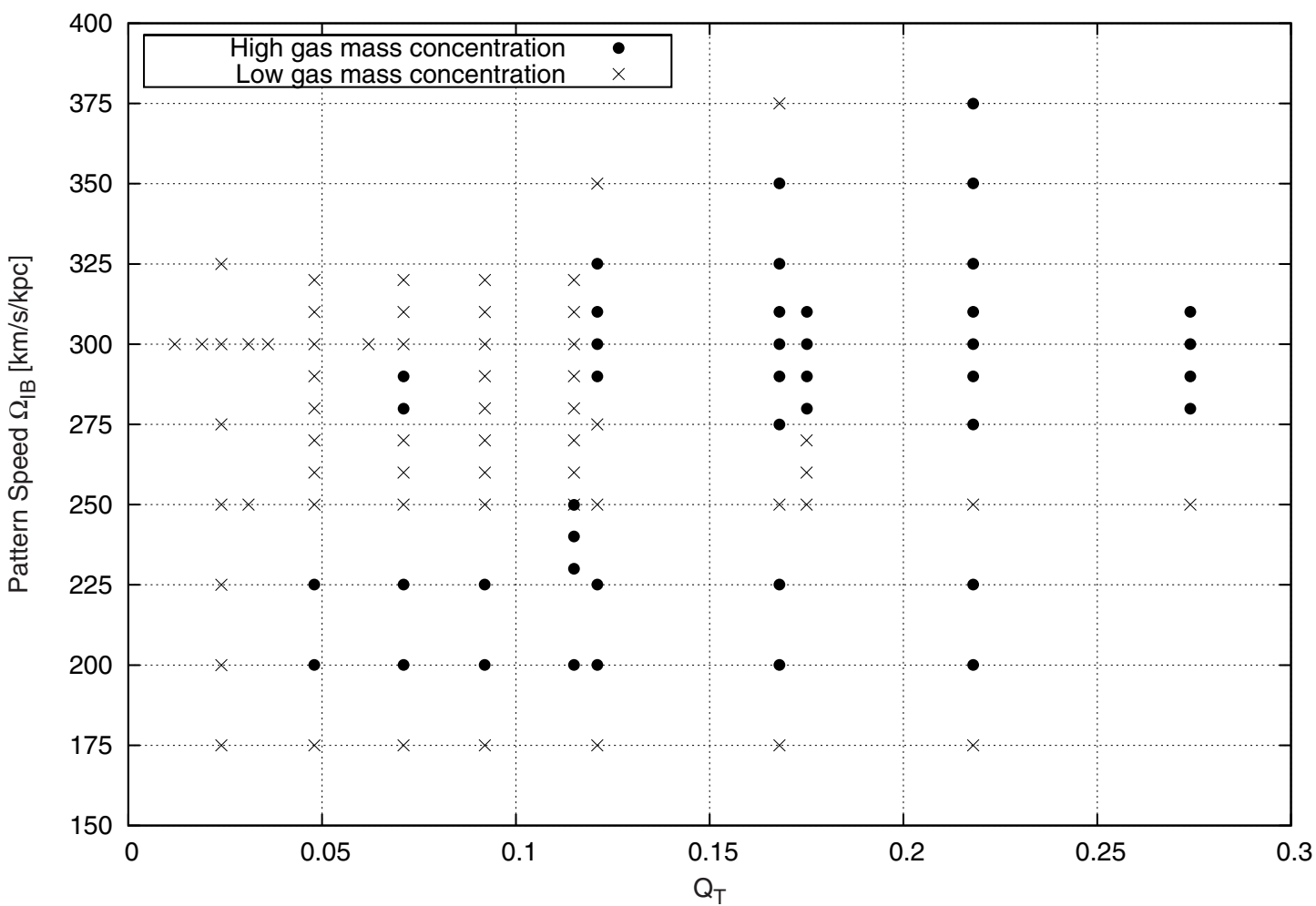

Figure 9. Gas mass concentration in our numerical results of the small inner bar models. The horizontal and vertical axes are $Q_{T}$ and $\Omega_{\mathrm{IB}}$, respectively. The high gas mass concentration models are shown by the filled circles, while the low gas mass concentration models are shown by the crosses.

gas ring. In this simulation, as a result of the fragmentation, the nuclear ring is disrupted. This is not consistent with the CMZ in our Galaxy.

We have shown a large amount of gas concentration to the Galactic center, by performing two-dimensional hydrodynamical simulations with various inner bar parameters (the size of the semimajor axis, mass, axial ratio, and the pattern speed of the inner bar). We have performed simulations for inner bars with $\Omega_{\mathrm{IB}} \approx(\Omega-\kappa / 2)_{\max }$, since this pattern speed is consistent with the $N$-body simulation results (Friedli \& Martinet 1993; Rautiainen et al. 2002). We have also performed simulations by changing the pattern speed of the inner bar for the small inner bar models to investigate the effect of the pattern speed on the mass inflow rate. We have assumed the two sizes of the semimajor axis of the inner bar, $200 \mathrm{pc}$, and $600 \mathrm{pc}$. We have found the high gas mass concentration in both sizes of the inner bar.

In the small inner bar models, the high gas mass concentration occurs for certain ranges of $Q_{T}$ and $\Omega_{\mathrm{IB}}$. In the models with $Q_{T} \gtrsim 0.12$, the second mass inflow to the Galactic center occurs for $\Omega_{\mathrm{IB}} \approx(\Omega-\kappa / 2)_{\max }$. However, in models with $Q_{T} \lesssim 0.12$, the second mass inflow does not occur for $\Omega_{\mathrm{IB}} \approx(\Omega-\kappa / 2)_{\max }$. For $0.05 \lesssim Q_{T} \lesssim 0.12$, the second mass inflow occurs for $\Omega_{\mathrm{IB}} \sim 225 \mathrm{~km} \mathrm{~s}^{-1} \mathrm{kpc}^{-1}$. Thus, the high gas mass concentration cases for the small inner bar models are divided into two cases:

1. $0.05 \lesssim Q_{T} \lesssim 0.12$ and $\Omega_{\mathrm{IB}} \sim 225 \mathrm{~km} \mathrm{~s}^{-1} \mathrm{kpc}^{-1}$;

2. $Q_{T} \gtrsim 0.12$, and $\Omega_{\mathrm{IB}} \approx(\Omega-\kappa / 2)_{\max }$ or $\Omega_{\mathrm{IB}} \sim$ $225 \mathrm{~km} \mathrm{~s}^{-1} \mathrm{kpc}^{-1}$.

One exception is the model S24, in which high gas mass concentration occurs for both $\Omega_{\mathrm{IB}} \approx(\Omega-\kappa / 2)_{\max }$ and $\Omega_{\mathrm{IB}} \sim$ $225 \mathrm{~km} \mathrm{~s}^{-1} \mathrm{kpc}^{-1}$ in spite of $0.05 \lesssim Q_{T} \lesssim 0.12$. These results are summarized in Figure 9. In this figure, the results of the model S25 $\left(\Omega_{\mathrm{IB}}=250 \mathrm{~km} \mathrm{~s}^{-1} \mathrm{kpc}^{-1}\right)$ and the model S41 $\left(\Omega_{\mathrm{IB}}=250 \mathrm{~km} \mathrm{~s}^{-1} \mathrm{kpc}^{-1}\right)$ occupy the same point at
$\left(Q_{T}, \Omega_{\mathrm{IB}}\right)=(0.115,250)$. The high gas mass concentration occurs in the model S25 $\left(\Omega_{\mathrm{IB}}=250 \mathrm{~km} \mathrm{~s}^{-1} \mathrm{kpc}^{-1}\right)$, while it does not occur in the model S41 $\left(\Omega_{\mathrm{IB}}=250 \mathrm{~km} \mathrm{~s}^{-1} \mathrm{kpc}^{-1}\right)$.

The second mass inflow rates change periodically in the models which are denoted by asterisks in Table 4 (see also the lower panel of Figure 6). These periodic changes imply that the second mass inflow is a resonance phenomenon between the outer bar and the inner bar, since the second mass inflow rate increases with the time intervals which are roughly the figure rotation period of the inner bar measured in the rotational frame of the outer bar.

The high gas mass concentration cases in the small inner bars models are consistent with observations in our Galaxy. In these models, a nuclear gas disk forms. Its size and mass are $R \lesssim 15 \mathrm{pc}$ and $\sim 10^{7} M_{\odot}$, respectively. Interestingly, the size of the nuclear gas disk is very close to the location of the Arches cluster and the Quintuplet cluster. Moreover, the nuclear gas disk is massive enough to form these star clusters (we discuss this point in Section 4.2). Kinematics of gas induced by the inner bar is consistent with the molecular gas observations (we discuss this point in Section 4.3). On the other hand, in the small inner bar models with $Q_{T}<0.05$, the inner bar does not highly enhance mass inflow to the galactic center. Hence, the inner bar in our Galaxy is $Q_{T} \gtrsim 0.05$, if mass supply to the Galactic center is due to the inner bar.

There is a difference between the size of the small inner bar models and the inner bar reported by Nishiyama et al. (2005, 2006). Nishiyama et al. $(2005,2006)$ trace the ridge of the distribution of red clump stars but do not show the profile of the gravitational potential of the inner bar. Our numerical results are consistent with their report, if the nonaxisymmetric component of the gravitational potential of the inner bar is small beyond $R=200 \mathrm{pc}$. 
The large inner bars in our models are not consistent with observations in our Galaxy, if mass supply to the Galactic center is caused by the large inner bar. In the large inner bar models with $Q_{T} \gtrsim 0.11$, high gas mass concentration occurs and the $200 \mathrm{pc}$ gas ring is destroyed. This does not correspond to our Galaxy. In the models with $Q_{T}<0.11$, the inner bar does not induce a large mass inflow to the Galactic center. From these results, the large inner bar is unlikely to be the case in the Galaxy, if mass supply to the Galactic center is due to an inner bar.

It is observed that the velocity dispersion of gas clouds in the central region of the Galaxy is higher than that in the Galactic disk (Rohlfs \& Kreitschmann 1987). Englmaier \& Gerhard (1997) show that the gas flow can change drastically when the sound speed is changed, since existence and strengths of shocks depend on $c_{s}$. In order to confirm the effect of the sound speed on the mass inflow, we try a test calculation in which the inner bar parameters are the same as the model S33 $\left(\Omega_{\mathrm{IB}}=300 \mathrm{~km} \mathrm{~s}^{-1} \mathrm{kpc}^{-1}\right)$ and the artificial radial profile of $c_{s}$, which rises from $\approx 10 \mathrm{~km} \mathrm{~s}^{-1}$ at the inner edge of the $200 \mathrm{pc}$ gas ring to $20 \mathrm{~km} \mathrm{~s}^{-1}$ at the center, is assumed. The straight shocks in the inner bar become weaker and the mass inflow rate becomes smaller. We will study the effect of the sound speed on the gas flow further in a future work considering a realistic cooling and heating process.

\subsection{Evolution of the Nuclear Gas Disk}

We have shown that small massive gas disks form in the small inner bar models for $Q_{T} \gtrsim 0.05$ and their size are $\sim 15 \mathrm{pc}$. It is interesting to study the self-gravitational instability of the nuclear gas disk. In an axisymmetric uniform thin gas disk, the dispersion relation of the small radial density perturbation in the axisymmetric mode is

$$
\omega^{2}=c_{s}^{2} k^{2}-2 \pi G \Sigma|k|+\kappa^{2},
$$

where $\omega$ is the frequency of the perturbation, $c_{s}$ is the sound speed of gas, $k$ is the wave number of the perturbation, $G$ is the gravitational constant, $\Sigma$ is the surface density of the thin disk, and $\kappa$ is the epicyclic frequency (Binney \& Tremaine 1987). From the dispersion relation, the density perturbation can grow if

$$
Q \equiv \frac{c_{s} \kappa}{\pi G \Sigma} \lesssim 1,
$$

where $Q$ is the Toomre $Q$-value. We define $\Sigma_{\text {crit }}$ as the surface density for $Q=1$,

$$
\Sigma_{\text {crit }}(R)=\frac{c_{s}(R) \kappa(R)}{\pi G},
$$

which may be the minimum surface density for the gravitational instability. Using $\Sigma_{\text {crit }}$, we define $M_{\text {crit }}$ as

$$
M_{\text {crit }}(R)=\int_{0}^{R} 2 \pi R^{\prime} \Sigma_{\text {crit }}\left(R^{\prime}\right) d R^{\prime} .
$$

$M_{\text {crit }}(R)$ may be a measure of the gravitational instability of the disk. In the central several tens parsecs of the galaxy, there is evidence for strong magnetic fields (e.g., Chuss et al. 2003). Magnetic fields have an important role in the gravitational stability of the disk. To consider the effect of magnetic fields in the linear analysis, we assume a simple configuration of magnetic fields, since it is observationally unclear. We assume that magnetic fields are parallel to the disk and homogeneous, $\boldsymbol{B}=B_{0} \boldsymbol{e}_{\boldsymbol{\phi}}$, where $B_{0}$ is the strength of magnetic fields and $\boldsymbol{e}_{\boldsymbol{\phi}}$ is the base vector of the azimuth. Fan \& Lou (1997) derived the dispersion relation

$$
\omega^{2}=\left(c_{s}^{2}+c_{A}^{2}\right) k^{2}-2 \pi G \Sigma|k|+k^{2}
$$

for this configuration, where $c_{A}$ is the Alfvén velocity,

$$
c_{A}=\sqrt{\frac{B^{2}}{4 \pi \rho}} .
$$

We use this dispersion relation for our analysis. We assume that gas clumps are formed from perturbations with the largest growth rate. The wavelength of the density perturbation with the largest growth rate is given by

$$
\lambda_{\max }=\frac{2 \pi}{k_{\max }}=\frac{2 c_{\mathrm{eff}}^{2}}{G \Sigma_{\mathrm{crit}}}=\frac{2 \pi c_{\mathrm{eff}}(R)}{\kappa(R)},
$$

where $c_{\text {eff }} \equiv \sqrt{c_{s}^{2}+c_{A}^{2}}$. The gas clump mass is estimated as

$$
M_{\text {clump }}=\pi\left(\frac{\lambda_{\max }}{2}\right)^{2} \Sigma_{\text {crit }}=\frac{\pi^{2} c_{\mathrm{eff}}^{3}(R)}{G \kappa(R)} .
$$

Application of these results to our numerical results show that strong magnetic fields, which are comparable with the strongest magnetic fields observed in the Galactic central region, enable massive gas clumps to grow and these are comparable to the mass of the young massive star clusters in the Galactic center. Figure 10 shows the result of the application for the model S33 $\left(\Omega_{\mathrm{IB}}=300 \mathrm{~km} \mathrm{~s}^{-1} \mathrm{kpc}^{-1}\right)$, which is one of the high gas mass concentration cases in the small inner bar models. In this figure, we assume that the gas in the nuclear gas disk sufficiently cools down to $T=100 \mathrm{~K}\left(c_{s} \approx 1 \mathrm{~km} \mathrm{~s}^{-1}\right)$. From this figure, the nuclear gas disk becomes gravitationally unstable after $t=300 \mathrm{Myr}$, if the effect of magnetic fields is very weak. The mass of the disk is $6.7 \times 10^{5} M_{\odot}$ at that time. The mass of the gas clumps is $100-300 M_{\odot}$ from Equation (10). If $B_{0}=$ $1 \mathrm{mG}$, the disk becomes unstable after $t=450 \mathrm{Myr}$. The mass of the disk is $2.9 \times 10^{6} M_{\odot}$ at that time. The mass of the gas clumps is $(1.0-3.0) \times 10^{4} M_{\odot}$. This mass is comparable to that of the young massive star clusters in the Galactic center.

Massive gas clumps can be formed even in the nonmagnetic case. To investigate the nonlinear evolution of the nuclear gas disk in the nonmagnetic case, we perform very high resolution hydrodynamical simulations in D. Namekata et al. (2009, in preparation, hereafter Paper II). In Paper II, we show that many massive compact gas clumps are formed by gravitational instability of the cooling gas disk in the nonmagnetic case. Typical mass and size of the clumps are several $10^{3} M_{\odot}$ to $10^{4} M_{\odot}$ and less than a few parsecs, respectively. The largest gas clumps have a mass of $\sim 10^{5} M_{\odot}$. This is much larger than $100-300 M_{\odot}$. This is because small gas clumps, which are formed rapidly from the growth of density perturbation in the cooling disk, collide each other and merge into more massive clumps. The Arches and Quintuplet clusters have a mass of $\sim 10^{4} M_{\odot}$ and a size of $<1 \mathrm{pc}$. If we assume a star formation efficiency of $\sim 0.1$, these clusters can be formed from the gas clump of mass $\sim 10^{5} M_{\odot}$, which is comparable to the largest gas clumps in our numerical results of Paper II.

\subsection{Longitude-Velocity Diagrams of Gas Flow in the Nested Bars}

We make longitude-velocity $(l-v)$ diagrams from our numerical results with the following two aims. One is to compare our numerical results with observations in our Galaxy. 
Table 5

High Gas Mass Concentration Cases in the Large Inner Bar Models

\begin{tabular}{|c|c|c|c|c|c|c|c|c|c|c|c|c|c|c|c|}
\hline Model Name & L41 & $\mathrm{L} 42$ & L43 & L31 & L32 & L33 & L34 & L35 & L22 & $\mathrm{L} 23$ & $\mathrm{~L} 24$ & L25 & L13 & L14 & L15 \\
\hline & $1.9 \mathrm{E}+5$ & $1.7 \mathrm{E}+8^{\mathrm{a}}$ & $3.2 \mathrm{E}+8^{\mathrm{a}}$ & $1.8 \mathrm{E}+5$ & $1.7 \mathrm{E}+5$ & $2.1 \mathrm{E}+8^{\mathrm{a}}$ & $6.6 \mathrm{E}+7^{\mathrm{a}}$ & $4.4 \mathrm{E}+8^{\mathrm{a}}$ & $1.7 \mathrm{E}+5$ & $1.9 \mathrm{E}+5$ & $2.6 \mathrm{E}+5$ & $2.6 \mathrm{E}+8^{\mathrm{a}}$ & $1.1 \mathrm{E}+5$ & $1.2 \mathrm{E}+5$ & $1.6 \mathrm{E}+5$ \\
\hline
\end{tabular}

Note. ${ }^{\text {a }}$ The models in which $M_{20}(t=500 \mathrm{Myr})>M_{\star}(<20 \mathrm{pc}) \approx 2 \times 10^{7} M_{\odot}$.
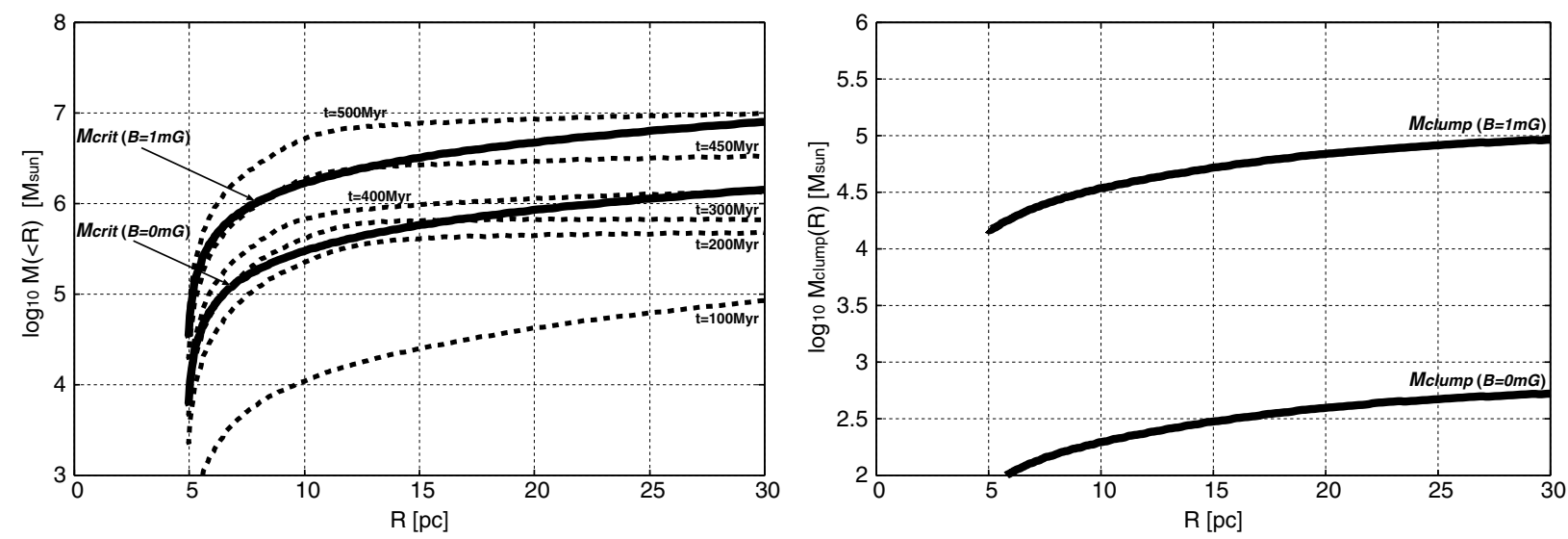

Figure 10. Left: time evolution of $M(<R)$ of the model S33 $\left(300 \mathrm{~km} \mathrm{~s}^{-1} \mathrm{kpc}^{-1}\right)$ and the critical disk mass $M_{\text {crit }}(R) . M(<R)$ at each time are shown by dashed lines, while $M_{\text {cirt }}(R)$ are shown by two solid lines for the case of $B_{0}=0$ and $1 \mathrm{mG}$. Right: the mass of the gas clump $M_{\text {clump }}$ given by Equation (10) at each radius.
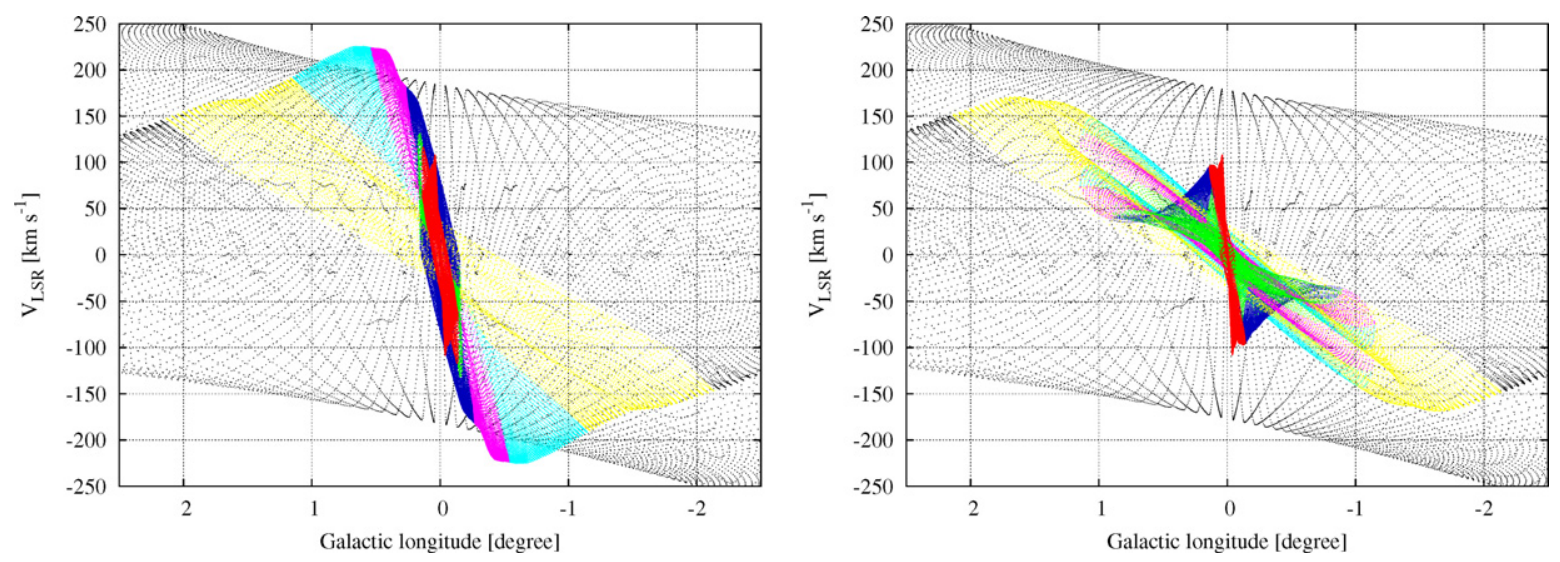

Figure 11. Longitude-velocity diagram of the model S33 $\left(\Omega_{\mathrm{IB}}=300 \mathrm{~km} \mathrm{~s}^{-1} \mathrm{kpc}^{-1}\right)$. The horizontal and vertical axes represent Galactic longitude $\left({ }^{\circ}\right)$ and line-of-sight velocity $\left(\mathrm{km} \mathrm{s}^{-1}\right)$, respectively. The left panel shows the $l-v$ diagram for $\Delta \theta^{\prime}=0^{\circ}(t=489 \mathrm{Myr})$. The right panel shows the $l-v$ diagram for $\Delta \theta^{\prime}=90^{\circ}(t=495 \mathrm{Myr})$. Red points: the nuclear gas disk component; green points: the straight shocks component; blue points: the inner void component, which corresponds to low-density regions within the elliptical gas ring; purple points: the elliptical gas ring component; aqua points: the outer void component, which corresponds to low-density regions between the elliptical ring and the 200 pc gas ring; yellow points: the $200 \mathrm{pc}$ gas ring component; black points: the region of $R>300 \mathrm{pc}$.

(A color version of this figure is available in the online journal.)

Another is to show that characteristic features of gas motion induced by the inner bar can be evidence of inner bars in external galaxies.

Figure 11 shows the $l-v$ diagrams of the model S33 $\left(\Omega_{\mathrm{IB}}=\right.$ $300 \mathrm{~km} \mathrm{~s}^{-1} \mathrm{kpc}^{-1}$ ), which is one of the high gas mass concentration cases in the small inner bar models, for $\Delta \theta^{\prime}=0^{\circ}$ and $90^{\circ}$, where $\Delta \theta^{\prime}$ is the angle between the direction of the inner bar and the Sun-Galactic center line. In the diagram, we assume that the outer bar is inclined at an angle of $20^{\circ}$ with respect to the Sun-Galactic center line, the distance of the Sun from the Galactic center is $8 \mathrm{kpc}$, and the circular velocity of the Sun is $220 \mathrm{~km} \mathrm{~s}^{-1}$. These assumptions are based on the results of Bissantz et al. (2003). In this figure, we classify the gas components of the results into seven groups by colors (the detail of the classification is described in the caption of Figure 11) according to the property of gas motion. The nuclear gas disk component is shown by the red points in Figure 11. The nuclear gas disk component in Figure 11 is weakly dependent on $\Delta \theta^{\prime}$, since circular motions dominate in the disk. The straight shocks component is shown by the green points in Figure 11. The feature of the straight shocks depends on $\Delta \theta^{\prime}$. When the inner bar is perpendicular to the outer bar, the straight shocks component is clearly distinguishable from the nuclear gas disk component and the $200 \mathrm{pc}$ gas ring component. The elliptical gas ring component is shown by the purple points in Figure 11. The feature of this ring strongly depends on $\Delta \theta^{\prime}$, since it is elongated along the inner bar.

There are many observational studies on gas distribution and kinematics in the central region of our Galaxy. Stark et al. (2004) give the $l-v$ diagram of highly excited rotational emission lines of $\mathrm{CO}(J=4-3$ and $J=7-6)$ in the central region of our Galaxy observed by AST/RO. They cover a range 
of $-1.2<l<2^{\circ}$. Their $l-v$ diagram traces high-density components of the molecular gas. Rodriguez-Fernandez et al. (2006) show the $l-v$ diagram of CO $(J=2-1)$ using the published data. Their $l-v$ diagram covers the same region as the $l-v$ diagram of Stark et al. (2004), but traces the diffuse molecular gas. It is known that there are two compact GMCs: the $20 \mathrm{~km} \mathrm{~s}^{-1}$ cloud and the $50 \mathrm{~km} \mathrm{~s}^{-1}$ cloud in the Galactic center region. The $20 \mathrm{~km} \mathrm{~s}^{-1}$ cloud is located at $R \lesssim 10 \mathrm{pc}$ from the center in the projection. It has a total mass of $\sim 3 \times 10^{5} M_{\odot}$ and radial velocities in the range of $\sim 5-25 \mathrm{~km} \mathrm{~s}^{-1}$. The $50 \mathrm{~km} \mathrm{~s}^{-1}$ cloud has a mass of $\sim 10^{5} M_{\odot}$ (Mezger et al. 1996). The positions of these GMCs in the $l-v$ diagram are shown in Nagayama et al. (2007). Oka et al. (2007) give the $l-v$ diagram of a highly excited rotational emission line of $\mathrm{CO}(J=3-2)$ with high resolution from $l=-0.2$ to 0.1 . They show that there is a pair of high-velocity emission (they are named $\mathrm{CND}^{+}$and $\mathrm{CND}^{-}$in their paper) within $0.05 \approx 6.5 \mathrm{pc}$ from $\mathrm{Sgr} \mathrm{A}^{*}$. The line-of-sight velocity of $\mathrm{CND}^{+}$and $\mathrm{CND}^{-}$is $50-100 \mathrm{~km} \mathrm{~s}^{-1}$ and -50 to $-120 \mathrm{~km} \mathrm{~s}^{-1}$, respectively.

Our numerical results are consistent with the observations in the central region of our Galaxy. The nuclear gas disk component in Figure 11 for $\Delta \theta^{\prime}=90^{\circ}$ is in the longitude range of $-0.2 \lesssim l \lesssim 0.2$ and in the velocity range of $-100 \mathrm{kms}^{-1} \lesssim v \lesssim 100 \mathrm{~km} \mathrm{~s}^{-1}$. This is the same range of the most inner $x_{2}$ orbit as shown in Stark et al. (2004). The velocity range of the nuclear gas disk agrees with that of the CND. The similar agreement between the nuclear gas disk component and the CND is also found in the $l-v$ diagram of RodriguezFernandez et al. (2006) and Oka et al. (2007). The 20 and $50 \mathrm{~km} \mathrm{~s}^{-1}$ clouds lie in the same region as the nuclear gas disk in the $l-v$ diagram (Nagayama et al. 2007). Thus, the nuclear gas disk component well corresponds to the observations. There are not clear high-velocity components corresponding to the elliptical gas ring component for $\Delta \theta^{\prime}=0^{\circ}$ in the $l-v$ diagrams of Stark et al. (2004) and Rodriguez-Fernandez et al. (2006). The other gas components in our $l-v$ diagrams occupy the same region in their $l-v$ diagrams. Thus, our $l-v$ diagrams for $\Delta \theta^{\prime}=90^{\circ}$ well corresponds to our Galaxy.

We compare our numerical results with the molecular gas observation in Maffei 2 . Our $l-v$ diagram at $\Delta \theta^{\prime}=90^{\circ}$ well corresponds to the $\mathrm{CO} p-v$ diagrams of the nuclear region of Maffei 2 (Meier et al. 2008). Meier et al. (2008) performed an observation of the nuclear region of Maffei 2 with a high spacial resolution with the OVRO and BIMA arrays and found a parallelogram feature and two intense features at both sides of the parallelogram feature in their $p-v$ diagrams. The parallelogram feature extends over $-5^{\prime \prime} \lesssim p \lesssim 15^{\prime \prime}$ and $-125 \mathrm{kms}^{-1} \lesssim v \lesssim 125 \mathrm{~km} \mathrm{~s}^{-1}$ in their diagrams. The two intense features are located at $(p, v) \approx\left(-15^{\prime \prime}, 50 \mathrm{kms}^{-1}\right)$ and $\left(20^{\prime \prime},-50 \mathrm{kms}^{-1}\right)$ in their diagram. They explain these features by simple linear orbits in their nuclear bar model. The nuclear gas disk component in our $l-v$ diagram corresponds to the parallelogram feature. The straight shocks component and the elliptical gas component in our $l-v$ diagram well correspond to the two intense features. Thus, our results strongly support their interpretation that Maffei 2 likely has a nuclear bar. We propose that the nuclear gas disk component, the straight shocks component, and the elliptical gas component are indirect evidence for an inner bar. Observation of molecular gas in the nuclear region of external barred galaxies with a high spacial resolution, e.g., ALMA, can give evidence of inner bars, even if they are hidden by a large amount of gas and dust.

\subsection{Important Role of a Central Mass Concentration}

We discuss the difference between our numerical results and those of Maciejewski et al. (2002). In our simulations, the massive nuclear gas disks are formed in the Galactic center. Formation of the nuclear gas disks is due to the straight shocks inside the inner bars. On the other hand, both such nuclear gas disks and straight shocks are not formed in Maciejewski et al. (2002), although they also simulated gas flow in a nested barred model.

We consider the central mass concentration as the main reason for the difference, since the major difference between our models and their model is the central mass concentration. We assumed the high central mass concentration that is modeled on the basis of the nuclear bulge profile given by Launhardt et al. (2002), while the central mass concentration in the model of Maciejewski et al. (2002) is low (see Figure 3 in Maciejewski \& Sparke 2000). It is shown that a high central mass concentration in a barred potential strongly affects orbital structure of stars and gas (Fukuda et al. 1998, 2000; Ann \& Thakur 2005). The central mass concentration tends to change the shape of the orbits of stars into rounder shapes at the nearer central region of the galaxy. When the galaxy has an inner bar, the shapes of the orbits are elongated at the radii which are comparable to the semimajor axis of the inner bar. In smaller radii, the shape of the orbits changes into circular orbits in the inner bar potential, if the central mass concentration is sufficiently high. Straight shocks may form if the shape of orbits rapidly varies as the radii become small, since gas collides at the region where the orbits are overcrowded and dissipates. Hence, a high central mass concentration is important for the formation of straight shocks and therefore the formation of nuclear gas disks. We conclude that the difference between our numerical results and those of Maciejewski et al. (2002) is mainly due to the difference in a central mass concentration. It is important to study a selfconsistent model of nested barred galaxies with high central mass concentrations and their stability.

\section{SUMMARY}

We summarize our study as follows:

1. We have performed two-dimensional hydrodynamical simulations to investigate the mass supply process by nested bars. We have assumed the gravitational potential model of our Galaxy, based on the Galaxy models of Bissantz et al. (2003) and the nuclear bulge profile given by Launhardt et al. (2002) adding an inner bar. We have assumed two cases of the size of the inner bar models: $a_{\mathrm{IB}}=200 \mathrm{pc}$ and $600 \mathrm{pc}$.

2. In the small inner bar models, a large amount of gas concentrates into the Galactic center for (1) $0.05 \lesssim Q_{T} \lesssim$ 0.12 and $\Omega_{\mathrm{IB}} \sim 225 \mathrm{~km} \mathrm{~s}^{-1} \mathrm{kpc}^{-1}$ and (2) $Q_{T} \gtrsim 0.12$ and, $\Omega_{\mathrm{IB}} \approx(\Omega-\kappa / 2)_{\max }$ or $\Omega_{\mathrm{IB}} \sim 225 \mathrm{~km} \mathrm{~s}^{-1} \mathrm{kpc}^{-1}$. The straight shocks are formed within the inner bar. This is partly due to that $Q_{T}$ in these models is high and partly due to that the central mass concentration in our models is high. The straight shocks sweep gas in the inner bar region. The gas trapped by the straight shocks falls into the Galactic center and then the nuclear gas disk is formed at the center. The size and mass of the nuclear gas disk are $\lesssim 15 \mathrm{pc}$ and $\sim 10^{7} M_{\odot}$, respectively.

3. In the large inner bar models, a large amount of gas concentrates into the Galactic center for $Q_{T}>0.11$. In the course of the gas concentration, the inner bar destroys 
the 200 pc gas ring. The destruction of the 200 pc gas ring is not consistent with the CMZ. We conclude that the inner bar of our Galaxy is not both large and strong, if the recent mass supply to the Galactic center is due to the inner bar of our Galaxy.

4. The high gas mass concentration cases in the small inner bar models well agree with the observed feature as follows. Extent and kinematics of the nuclear gas disk in our results are consistent with the observations of the molecular gas in the central region of our Galaxy. The size of the nuclear gas disk is very close to the location of the Arches cluster and the Quintuplet cluster, and its mass is enough to form these star clusters.

5. We have discussed the self-gravitational instability of the nuclear gas disk formed in our simulations. Assuming magnetic fields as strong as the observed one in the central tens parsecs of our Galaxy, the most rapid growing unstable mode corresponds to gas clumps which have comparable mass to the Arches and Quintuplet clusters. In the next paper, we will study the nonlinear evolution of massive nuclear gas disks.

6. We have shown the characteristic features in the $l-v$ diagram induced by the small inner bar. These features can be clues about the existence of inner bars in extra galaxies. They will be useful for future observation of central regions of galaxies, e.g., ALMA.

We thank Masayuki Fujimoto and Kazuo Sorai for fruitful discussions. This work has been supported in part by the Hokkaido University Grant Program for New Fusion of Extensive Research Fields, in part by grants-in-aid for Scientific Research $(14340058,19540233)$ of the Japan Society for the Promotion of Science.

\section{REFERENCES}

Alard, C. 2001, A\&A, 379, 44

Ann, H. B., \& Thakur, P. 2005, ApJ, 620, 197

Athanassoula, E. 1992, MNRAS, 259, 345

Binney, J., Gerhard, O., Stark, A., Bally, J., \& Uchida, K. 1991, MNRAS, 252, 210

Binney, J., \& Tremaine, S. 1987, in Galactic Dynamics, (Princeton, NJ: Princeton Univ. Press)

Bissantz, N., Englmaier, P., \& Gerhard, O. 2003, MNRAS, 340, 949

Buta, R., \& Crocker, D. 1993, ApJ, 105, 1344

Christopher, M., Scoville, N., Stolovy, S., \& Yun, M. 2005, ApJ, 622, 346

Chuss, D., Davidson, J., Dotson, J., Dowell, C., Hildebrand, R., Novak, G., \& Vaillancourt, J. 2003, ApJ, 599, 1116

Coil, A., \& Ho, P. 1999, ApJ, 513, 752

Colina, L., \& Wada, K. 2000, ApJ, 529, 845

Debattista, V., \& Shen, J. 2007, ApJ, 654, 127

Eisenhauer, F., Schödel, R., Genzel, R., Ott, T., Tecza, M., Abuter, R., Eckart, A., \& Alexander, T. 2003, ApJ, 597, 121

El-Zant, A., \& Shlosman, I. 2003, ApJ, 595, L41
Englmaier, P., \& Gerhard, O. 1997, MNRAS, 287, 57

Englmaier, P., \& Shlosman, I. 2000, ApJ, 528, 677

Englmaier, P., \& Shlosman, I. 2004, ApJ, 617, L115

Erwin, P. 2004, A\&A, 415, 941

Erwin, P., \& Sparke, L. 2002, AJ, 124, 65

Fan, Z., \& Lou, Y.-Q. 1997, MNRAS, 291, 91

Fathi, K., Storchi-Bergmann, T., Riffel, R., Winge, C., Axon, D., Robinson, A., Capetti, A., \& Marconi, A. 2006, ApJ, 641, L25

Ferrers, N. 1877, Q. J. Pure Appl. Math., 14, 1

Figer, D. 2003, IAU Symp. 212, A Massive Star Odyssey: From Main Sequence to Supernova, ed. K. A. van der Hucht, A. Herrero, \& C. Esteban (San Francisco, CA: ASP), 487

Friedli, D. 1996, A\&A, 312, 761

Friedli, D., \& Martinet, L. 1993, A\&A, 277, 27

Friedli, D., Wozniak, H., Rieke, M., Martinet, L., \& Bratschi, P. 1996, A\&AS, 118,461

Fukuda, H., Habe, A., \& Wada, K. 2000, ApJ, 529, 109

Fukuda, H., Wada, K., \& Habe, A. 1998, MNRAS, 295, 463

Heller, C., Shlosman, I., \& Athanassoula, E. 2006, ApJ, 657, 65

Heller, C., Shlosman, I., \& Englmaier, P. 2001, ApJ, 553, 661

Jungwiert, B., Combes, F., \& Axon, D. 1997, A\&AS, 125, 479

Launhardt, R., Zylka, R., \& Mezger, P. 2002, A\&A, 384, 112

Liou, M.-S., \& Steffen, C. 1993, J. Comput. Phys., 107, 23

Maciejewski, W., \& Sparke, L. 1997, ApJ, 484, 117

Maciejewski, W., \& Sparke, L. 2000, MNRAS, 313, 745

Maciejewski, W., Teuben, P., Sparke, L., \& Stone, J. 2002, MNRAS, 329, 502

Meier, D., Turner, J., \& Hurt, R. 2008, ApJ, 675, 281

Mezger, P. G., Duschl, W. J., \& Zylka, R. 1996, A\&A Rev., 7, 289

Mori, M., Ferrara, A., \& Madau, P. 2002, ApJ, 571, 40

Morris, M., \& Serabyn, E. 1996, ARA\&A, 34, 645

Nagayama, T., Omodaka, T., Handa, T., Iahak, H. B. H., Sawada, T., Miyaji, T., \& Koyama, Y. 2007, PASJ, 59, 869

Nishiyama, S., Nagata, T., \& IRSF/SIRIUS Team 2006, J. Phys. Conf. Ser., 54, 62

Nishiyama, S., et al. 2005, ApJ, 621, 105

Oka, T., Nagai, M., Kamegai, K., Tanaka, K., \& Kuboi, N. 2007, PASJ, 59, 15

Prieto, M., Maciejewski, W., \& Reunanen, J. 2005, AJ, 130, 1472

Radespiel, R., \& Kroll, N. 1995, J. Comput. Phys., 121, 66

Rattenbury, N. J., Mao, S., Sumi, T., \& Smith, M. C. 2007, MNRAS, 378, 1064

Rautiainen, P., Salo, H., \& Laurikainen, E. 2002, MNRAS, 337, 1233

Regan, M. W., \& Teuben, P. J. 2003, ApJ, 582, 723

Rodriguez-Fernandez, N., Combes, F., Martin-Pintado, J., Wilson, T., \& Apponi, A. 2006, A\&A, 455,963

Rohlfs, K., \& Kreitschmann, J. 1987, A\&A, 178, 95

Sawada, T., Hasegawa, T., Handa, T., \& Cohen, R. 2004, MNRAS, 349, 1167

Schinnerer, E., Böker, T., Emsellem, E., \& Downes, D. 2007, A\&A, 462, L27

Schinnerer, E., Böker, T., Emsellem, E., \& Lisenfeld, U. 2006, ApJ, 649, 181

Serabyn, E., \& Morris, M. 1995, Nature, 382, 15

Shaw, M., Combes, F., Axon, D., \& Wright, G. 1993, A\&A, 273, 31

Shen, J., \& Dibattista, V. P. 2007, arXiv:0711.0966v1

Shlosman, I., Frank, J., \& Begelman, M. 1989, Nature, 338, 45

Shlosman, I., \& Heller, C. 2002a, ApJ, 565, 921

Shlosman, I., \& Heller, C. 2002b, ApJ, 565, 921

Stanek, K., Udalski, A., Szymański, M., Kałuzny, J., Kubiak, M., Mateo, M., \& Krzenmiński, W. 1997, ApJ, 477, 163

Stark, A. A., Martin, C. L., Walsh, W. M., Xiao, K., Lane, A. P., \& Walker, C. K. 2004, ApJ, 614, L41

Wada, K., \& Norman, C. A. 2001, ApJ, 547, 172

Wozniak, H., Friedli, D., Martinet, L., Martin, P., \& Bratschi, P. 1995, A\&AS, 111,115 